\title{
Chemical Constituents and Pharmacological Activity of Agarwood and Aquilaria Plants
}

\author{
Shuai Wang ${ }^{1,2,+}{ }^{(0)}$, Zhangxin $\mathrm{Yu}^{3,4,+}$, Canhong Wang ${ }^{3,4}$, Chongming $\mathrm{Wu}^{5}$, Peng Guo ${ }^{5, *}$ \\ and Jianhe Wei $1,2,3,4, *$ \\ 1 Key Laboratory of Bioactive Substances and Resources Utilization of Chinese Herbal Medicine, \\ Institute of Medicinal Plant Development, Chinese Academy of Medical Sciences \& Peking Union \\ Medical College, Beijing 100193, China; zhuizhirun@163.com \\ 2 Ministry of Education \& National Engineering Laboratory for Breeding of Endangered Medicinal Materials, \\ Institute of Medicinal Plant Development, Chinese Academy of Medical Sciences \& Peking Union Medical \\ College, Beijing 100193, China \\ 3 Conservation and Development of Southern Medicine, Hainan Branch of the Institute of Medicinal \\ Plant Development, Chinese Academy of Medical Sciences \& Peking Union Medical College, Haikou 570311, \\ China; yu_xin712@163.com (Z.Y.); xinzhuangjianpo@163.com (C.W.) \\ 4 Key Laboratory of State Administration of Traditional Chinese Medicine for Agarwood Sustainable \\ Utilization, Hainan Branch of the Institute of Medicinal Plant Development, Chinese Academy of Medical \\ Sciences \& Peking Union Medical College, Haikou 570311, China \\ 5 Pharmacology and Toxicology Center, Institute of Medicinal Plant Development, Chinese Academy of \\ Medical Sciences \& Peking Union Medical College, Beijing 100193, China; cmwu@implad.ac.cn \\ * Correspondence: pguo@implad.ac.cn (P.G.); wjianh@263.net (J.W.); \\ Tel.: +86-010-5783-3235 (P.G.); +86-010-5783-3016 (J.W.) \\ + These authors contributed equally to this work.
}

Received: 10 January 2018; Accepted: 31 January 2018; Published: 7 February 2018

\begin{abstract}
Agarwood, a highly precious non-timber fragrant wood of Aquilaria spp. (Thymelaeaceae), has been widely used in traditional medicine, religious rites, and cultural activities. Due to the inflated demanding and depleted natural resources, the yields of agarwood collected from the wild are shrinking, and the price is constantly rising, which restricts agarwood scientific research and wide application. With the sustainable planting and management of agarwood applied, and especially the artificial-inducing methods being used in China and Southeast Asian countries, agarwood yields are increasing, and the price is becoming more reasonable. Under this condition, illuminating the scientific nature of traditional agarwood application and developing new products and drugs from agarwood have become vitally important. Recently, the phytochemical investigations have achieved fruitful results, and more than 300 compounds have been isolated, including numerous new compounds that might be the characteristic constituents with physiological action. However, no one has focused on the new compounds and presented a summary until now. Alongside phytochemical advances, bioactivity screening and pharmacological investigation have also made a certain progress. Therefore, this review discussed the new compounds isolated after 2010, and summarized the pharmacological progress on agarwood and Aquilaria plants.
\end{abstract}

Keywords: agarwood; Aquilaria plants; chemical constitutes; bioactive compounds; pharmacological function

\section{Introduction}

Agarwood, known as chenxiang in Chinese and called aloeswood, agalloch, eaglewood, jinkoh, gaharu, or kanankoh in different regions, is a highly valuable non-timber fragrant wood of Aquilaria spp. (Thymelaeaceae) [1-4]. There are 31 species of Aquilaria found worldwide in Indonesia, Malaysia, 
China, India, Philippines, Cambodia, Vietnam, Laos, Thailand, Papua New Guinea, and Singapore [5,6], among which 19 species can produce agarwood after being attacked by physical force [7,8], insects [9], or bacteria/fungi infection [10-12]. Agarwood is used for incense, perfume, traditional medicine, and other products in the world market. In traditional Chinese medicine, agarwood is used as a qi-regulating drug and carminative medicine to relieve gastric problems, coughs, rheumatism, and high fever. It can promote $q i$ circulation to relieve pain, warm the middle energizer to arrest vomiting, and regulate respiration to relieve asthma [13]. In traditional Arabian medicine, agarwood essential oil is used for aromatherapy. Simultaneously, agarwood has also been widely used for centuries as incense in Buddhist, Hindu, and Islamic ceremonies.

With the increasing demand for agarwood, the population of Aquilaria species is declining rapidly in the wild, and all species of Aquilaria have been placed on the Appendix II list of the Convention on International Trade in Endangered Species of Wild Fauna and Flora since 2004 [1]. In response to this situation, sustainable planting and management of agarwood with artificial methods are arising, and the agarwood yield is increasing. As a result, agarwood no longer needs to be obtained from wild natural resources, enabling its wider application and investigation, especially on pharmaceutical study. Based on the phytochemical studies, a number of new compounds have been isolated and identified from agarwood and Aquilaria plants. However, there is no literature concentrating on the new compounds, even though earlier literature have summarized the chemical constituents of agarwood and related plants $[6,14,15]$. Therefore, this review discussed the new compounds isolated after 2010, and summarized the pharmacological progress on agarwood and Aquilaria plants.

\section{Results and Discussion}

\subsection{New Compounds from Agarwood and Aquilaria Plants}

The chemical constitutes of agarwood originating from the genus Aquilaria, include 2-(2-phenylethyl)-4H-chromen-4-one derivatives, terpenoids, flavonoids etc., in which 2-(2-phenylethyl)-4H-chromen-4-one derivatives and sesquiterpenes are the two predominant constituents in agarwood. There have been 154 new compounds (Table 1) isolated from agarwood and genus Aquilaria trees since 132 compounds were summarized in June 2010 [14].

\subsubsection{2-(2-Phenylethyl)chromones}

In total, 88 new 2-(2-phenylethyl)chromone compounds (1-88) have been isolated from agarwood and genus Aquilaria plants (Figures 1-5). Yang et al. [16] carried out a bioassay-guided isolation strategy from $A$. sinensis, resulting in seven new 2-(2-phenylethl)chromone derivatives (1-7) and a new 2-(2-phenylethenyl)chromone (8) being obtained from an ethanol (EtOH) extract. The investigation of EtOH extract obtained another three 2-(2-phenylethl)chromones (9-11) $[17,18]$ and eight derivatives (12-19) from different fractions [19]. Liao et al. [20] reported seven new 2-(2-phenylethyl)chromone derivatives (20-26), including a chlorinated one (23) from the ethyl acetate (EtOAc) fraction of artificial agarwood (A. sinensis). The EtOAc fraction also contained three 2-(2-phenylethyl)chromones (27-29) [8] and four new bi-phenylethylchromones (30-33) [21]. A phytochemical investigation of a resinous wood (A. sinensis) led to the isolation of nine new 2-(2-phenylethyl)chromone derivatives, aquilarones A-I (34-42) from a chloroform $\left(\mathrm{CHCl}_{3}\right)$ fraction [22]. Liu et al. found four new 2-(2-phenylethyl)chromone derivatives (43-46) from Chinese agarwood produced via the whole-tree agarwood-inducing technique [23]. Huo et al. gained five new 2-(2-phenylethyl)chromone derivatives (47-51) [24] and sixteen dimeric 2-(2-phenylethyl)chromones (52-67) from the resinous wood of A. sinensis [25]. Liao et al. isolated thirteen 5,6,7,8-tetrahydro-2-(2-phenylethyl)chromones (68-80) [26] from the artificial agarwood of $A$. sinensis. Additionally, one 2-(2-phenylethyl)chromone compound (81) was isolated from the stem bark EtOH extract of $A$. sinensis [27].

"Qi-Nan" is regarded as the highest quality agarwood, valued for its mysterious oriental odor that can be smelt without burning, unlike other kinds of agarwood. The investigation of EtOH extract of 
high-quality Chinese agarwood "Qi-Nan" (A. sinensis) obtained seven new 2-(2-phenylethl)chromone derivatives (82-88) [28,29].<smiles>[R]c1ccc(C([R7])Cc2cc(=O)c3c([R])c([R])c([R])c([R])c3o2)c([R])c1[R]</smiles>

\begin{tabular}{|c|c|c|c|c|c|c|c|c|}
\hline NO. & $\mathbf{R}^{1}$ & $\mathbf{R}^{2}$ & $\mathbf{R}^{3}$ & $\mathbf{R}^{4}$ & $\mathbf{R}^{5}$ & $R^{6}$ & $\mathbf{R}^{7}$ & $\mathbf{R}^{8}$ \\
\hline 1 & $\mathrm{H}$ & $\mathrm{OH}$ & $\mathrm{OCH}_{3}$ & $\mathrm{H}$ & $\mathrm{H}$ & $\mathrm{H}$ & $\mathrm{OH}$ & $\mathrm{OCH}_{3}$ \\
\hline 2 & $\mathrm{H}$ & $\mathrm{OCH}_{3}$ & $\mathrm{OCH}_{3}$ & $\mathrm{H}$ & $\mathrm{H}$ & $\mathrm{H}$ & $\mathrm{OH}$ & $\mathrm{OCH}_{3}$ \\
\hline 3 & $\mathrm{H}$ & $\mathrm{OCH}_{3}$ & $\mathrm{OH}$ & $\mathrm{H}$ & $\mathrm{H}$ & $\mathrm{H}$ & $\mathrm{OH}$ & $\mathrm{OCH}_{3}$ \\
\hline 4 & $\mathrm{H}$ & $\mathrm{OCH}_{3}$ & $\mathrm{OCH}_{3}$ & $\mathrm{H}$ & $\mathrm{H}$ & $\mathrm{H}$ & $\mathrm{OCH}_{3}$ & $\mathrm{OH}$ \\
\hline 5 & $\mathrm{H}$ & $\mathrm{OH}$ & $\mathrm{OH}$ & $\mathrm{H}$ & $\mathrm{H}$ & $\mathrm{H}$ & $\mathrm{H}$ & $\mathrm{OCH}_{3}$ \\
\hline 6 & $\mathrm{H}$ & $\mathrm{OH}$ & $\mathrm{OCH}_{3}$ & $\mathrm{H}$ & $\mathrm{H}$ & $\mathrm{H}$ & $\mathrm{H}$ & $\mathrm{OH}$ \\
\hline 7 & $\mathrm{H}$ & $\mathrm{OH}$ & $\mathrm{H}$ & $\mathrm{OH}$ & $\mathrm{H}$ & $\mathrm{H}$ & $\mathrm{OH}$ & $\mathrm{OCH}_{3}$ \\
\hline 9 & $\mathrm{OH}$ & $\mathrm{OH}$ & $\mathrm{OH}$ & $\mathrm{OH}$ & $\mathrm{H}$ & $\mathrm{H}$ & $\mathrm{OH}$ & $\mathrm{OCH}_{3}$ \\
\hline 10 & $\mathrm{H}$ & $\mathrm{OH}$ & $\mathrm{H}$ & $\mathrm{Cl}$ & $\mathrm{H}$ & $\mathrm{H}$ & $\mathrm{H}$ & $\mathrm{H}$ \\
\hline 11 & $\mathrm{H}$ & $\mathrm{OH}$ & $\mathrm{H}$ & $\mathrm{Cl}$ & $\mathrm{H}$ & $\mathrm{H}$ & $\mathrm{H}$ & $\mathrm{OCH}_{3}$ \\
\hline 12 & $\mathrm{H}$ & $\mathrm{OCH}_{3}$ & $\mathrm{OH}$ & $\mathrm{H}$ & $\mathrm{H}$ & $\mathrm{H}$ & $\mathrm{H}$ & $\mathrm{OCH}_{3}$ \\
\hline 20 & $\mathrm{H}$ & $\mathrm{OH}$ & $\mathrm{OCH}_{3}$ & $\mathrm{H}$ & $\mathrm{H}$ & $\mathrm{H}$ & $\mathrm{H}$ & $\mathrm{OCH}_{3}$ \\
\hline 21 & $\mathrm{H}$ & $\mathrm{OH}$ & $\mathrm{H}$ & $\mathrm{H}$ & $\mathrm{H}$ & $\mathrm{H}$ & $\mathrm{OCH}_{3}$ & $\mathrm{OCH}_{3}$ \\
\hline 22 & $\mathrm{H}$ & $\mathrm{OH}$ & $\mathrm{H}$ & $\mathrm{OH}$ & $\mathrm{H}$ & $\mathrm{H}$ & $\mathrm{H}$ & $\mathrm{OCH}_{3}$ \\
\hline 23 & $\mathrm{H}$ & $\mathrm{OH}$ & $\mathrm{H}$ & $\mathrm{Cl}$ & $\mathrm{H}$ & $\mathrm{H}$ & $\mathrm{OH}$ & $\mathrm{OCH}_{3}$ \\
\hline 24 & $\mathrm{OCH}_{3}$ & $\mathrm{OH}$ & $\mathrm{H}$ & $\mathrm{H}$ & $\mathrm{H}$ & $\mathrm{H}$ & $\mathrm{OH}$ & $\mathrm{OCH}_{3}$ \\
\hline 25 & $\mathrm{H}$ & $\mathrm{OCH}_{3}$ & $\mathrm{OCH}_{3}$ & $\mathrm{H}$ & $\alpha-\mathrm{OH}$ & $\mathrm{H}$ & $\mathrm{H}$ & $\mathrm{H}$ \\
\hline 26 & $\mathrm{H}$ & $\mathrm{OCH}_{3}$ & $\mathrm{OCH}_{3}$ & $\mathrm{H}$ & $\beta-\mathrm{OH}$ & $\mathrm{H}$ & $\mathrm{H}$ & $\mathrm{H}$ \\
\hline 27 & $\mathrm{H}$ & $\mathrm{OCH}_{3}$ & $\mathrm{H}$ & $\mathrm{H}$ & $\mathrm{H}$ & $\mathrm{H}$ & $\mathrm{OH}$ & $\mathrm{OCH}_{3}$ \\
\hline 28 & $\mathrm{OH}$ & $\mathrm{OCH}_{3}$ & $\mathrm{H}$ & $\mathrm{H}$ & $\mathrm{H}$ & $\mathrm{H}$ & $\mathrm{OH}$ & $\mathrm{OCH}_{3}$ \\
\hline 40 & $\mathrm{H}$ & $\mathrm{OH}$ & $\mathrm{OCH}_{3}$ & $\mathrm{H}$ & $\mathrm{H}$ & $\mathrm{H}$ & $\mathrm{OCH}_{3}$ & $\mathrm{OH}$ \\
\hline 41 & $\mathrm{H}$ & $\mathrm{OCH}_{3}$ & $\mathrm{H}$ & $\mathrm{H}$ & $\mathrm{H}$ & $\mathrm{H}$ & $\mathrm{H}$ & $\mathrm{OH}$ \\
\hline 42 & $\mathrm{H}$ & $\mathrm{OH}$ & $\mathrm{H}$ & $\mathrm{H}$ & $\mathrm{H}$ & $\mathrm{H}$ & $\mathrm{OH}$ & $\mathrm{OCH}_{3}$ \\
\hline 43 & $\mathrm{OH}$ & $\mathrm{H}$ & $\mathrm{OCH}_{3}$ & $\mathrm{H}$ & $\mathrm{H}$ & $\mathrm{H}$ & $\mathrm{H}$ & $\mathrm{OCH}_{3}$ \\
\hline 44 & $\mathrm{OH}$ & $\mathrm{OCH}_{3}$ & $\mathrm{H}$ & $\mathrm{OH}$ & $\mathrm{H}$ & $\mathrm{H}$ & $\mathrm{H}$ & $\mathrm{H}$ \\
\hline 46 & $\mathrm{H}$ & $\mathrm{OCH}_{3}$ & $\mathrm{H}$ & $\mathrm{H}$ & $\mathrm{H}$ & $\mathrm{OH}$ & $\mathrm{OH}$ & $\mathrm{OH}$ \\
\hline 47 & $\mathrm{OH}$ & $\mathrm{OCH}_{3}$ & $\mathrm{OCH}_{3}$ & $\mathrm{H}$ & $\mathrm{H}$ & $\mathrm{H}$ & $\mathrm{H}$ & $\mathrm{OCH}_{3}$ \\
\hline 81 & $\mathrm{H}$ & $\mathrm{OCH}_{3}$ & $\mathrm{OH}$ & $\mathrm{H}$ & $\mathrm{H}$ & $\mathrm{H}$ & $\mathrm{H}$ & $\mathrm{H}$ \\
\hline 82 & $\mathrm{H}$ & $\mathrm{H}$ & $\mathrm{H}$ & $\mathrm{H}$ & $\mathrm{H}$ & $\mathrm{H}$ & $\mathrm{OH}$ & $\mathrm{OCH}_{3}$ \\
\hline 83 & $\mathrm{H}$ & $\mathrm{H}$ & $\mathrm{H}$ & $\mathrm{H}$ & $\mathrm{H}$ & $\mathrm{H}$ & $\mathrm{OCH}_{3}$ & $\mathrm{OH}$ \\
\hline 84 & $\mathrm{H}$ & $\mathrm{H}$ & $\mathrm{H}$ & $\mathrm{H}$ & $\mathrm{H}$ & $\mathrm{OH}$ & $\mathrm{H}$ & $\mathrm{OCH}_{3}$ \\
\hline 85 & $\mathrm{H}$ & $\mathrm{H}$ & $\mathrm{H}$ & $\mathrm{H}$ & $\mathrm{H}$ & $\mathrm{H}$ & $\mathrm{H}$ & $\mathrm{OH}$ \\
\hline 86 & $\mathrm{H}$ & $\mathrm{H}$ & $\mathrm{H}$ & $\mathrm{H}$ & $\mathrm{H}$ & $\mathrm{H}$ & $\mathrm{OH}$ & $\mathrm{H}$ \\
\hline 87 & $\mathrm{H}$ & $\mathrm{H}$ & $\mathrm{H}$ & $\mathrm{H}$ & $\mathrm{H}$ & $\mathrm{OH}$ & $\mathrm{H}$ & $\mathrm{H}$ \\
\hline 88 & $\mathrm{H}$ & $\mathrm{H}$ & $\mathrm{H}$ & $\mathrm{H}$ & $\mathrm{OH}$ & $\mathrm{H}$ & $\mathrm{H}$ & $\mathrm{H}$ \\
\hline
\end{tabular}

Figure 1. Structures of chromones identified in agarwood.<smiles>COc1cc(/C=C/c2cc(=O)c3cc(O)ccc3o2)ccc1O</smiles>

8

Figure 2. Structure of 2-(2-phenylethenyl)chromone identified in agarwood. 
<smiles>[R]c1ccc(CCc2cc(=O)c3c(o2)C([R])C([R])C([R])C3[R])cc1[R]</smiles>

\begin{tabular}{|c|c|c|c|c|c|c|}
\hline NO. & $\mathrm{R}^{1}$ & $\mathbf{R}^{2}$ & $\mathbf{R}^{3}$ & $\mathbf{R}^{4}$ & $\mathbf{R}^{5}$ & $R^{6}$ \\
\hline 16 & $\alpha-\mathrm{OH}$ & $\beta-\mathrm{OH}$ & $\beta-\mathrm{OH}$ & $\alpha-\mathrm{Cl}$ & $\mathrm{H}$ & $\mathrm{OCH}_{3}$ \\
\hline 17 & $\alpha-\mathrm{OH}$ & $\beta-\mathrm{OH}$ & $\beta-\mathrm{OH}$ & $\alpha-\mathrm{Cl}$ & $\mathrm{OH}$ & $\mathrm{OCH}_{3}$ \\
\hline 18 & $\alpha-\mathrm{OH}$ & $\alpha-\mathrm{OH}$ & $\beta-\mathrm{OH}$ & $\mathrm{H}$ & $\mathrm{H}$ & $\mathrm{H}$ \\
\hline 19 & $\alpha-\mathrm{OH}$ & $\alpha-\mathrm{OH}$ & $\beta-\mathrm{OH}$ & $\mathrm{H}$ & $\mathrm{H}$ & $\mathrm{OCH}_{3}$ \\
\hline 34 & $\alpha-\mathrm{OH}$ & $\alpha-\mathrm{OH}$ & $\alpha-\mathrm{OH}$ & $\beta-\mathrm{OH}$ & $\mathrm{OH}$ & $\mathrm{OCH}_{3}$ \\
\hline 35 & $\alpha-\mathrm{OH}$ & $\alpha-\mathrm{OH}$ & $\alpha-\mathrm{OH}$ & $\beta-\mathrm{OH}$ & $\mathrm{H}$ & $\mathrm{H}$ \\
\hline 36 & $\alpha-\mathrm{OH}$ & $\alpha-\mathrm{OH}$ & $\alpha-\mathrm{OH}$ & $\beta-\mathrm{OH}$ & $\mathrm{H}$ & $\mathrm{OCH}_{3}$ \\
\hline 37 & $\alpha-\mathrm{OH}$ & $\beta-\mathrm{OH}$ & $\alpha-\mathrm{OH}$ & $\beta-\mathrm{OH}$ & $\mathrm{OH}$ & $\mathrm{OCH}_{3}$ \\
\hline 38 & $\alpha-\mathrm{OH}$ & $\beta-\mathrm{OH}$ & $\beta-\mathrm{OH}$ & $\alpha-\mathrm{OH}$ & $\mathrm{OH}$ & $\mathrm{OCH}_{3}$ \\
\hline 39 & $\alpha-\mathrm{OH}$ & $\beta-\mathrm{OH}$ & $\beta-\mathrm{OH}$ & $\alpha-\mathrm{OH}$ & $\mathrm{H}$ & $\mathrm{OH}$ \\
\hline 48 & $\beta-\mathrm{OH}$ & $\beta-\mathrm{OH}$ & $\beta-\mathrm{OH}$ & $\alpha-\mathrm{Cl}$ & $\mathrm{H}$ & $\mathrm{OCH}_{3}$ \\
\hline 49 & $\alpha-\mathrm{OH}$ & $\alpha-\mathrm{OH}$ & $\alpha-\mathrm{OH}$ & $\alpha-\mathrm{Cl}$ & $\mathrm{H}$ & $\mathrm{H}$ \\
\hline 50 & $\beta-\mathrm{OH}$ & $\beta-\mathrm{OH}$ & $\beta-\mathrm{OH}$ & $\beta-\mathrm{Cl}$ & $\mathrm{H}$ & $\mathrm{OCH}_{3}$ \\
\hline 51 & $\beta-\mathrm{OH}$ & $\beta-\mathrm{OH}$ & $\beta-\mathrm{OH}$ & $\mathrm{H}$ & $\mathrm{OCH}_{3}$ & $\mathrm{OH}$ \\
\hline 68 & $\alpha-\mathrm{OCH}_{3}$ & $\beta-\mathrm{OH}$ & $\beta-\mathrm{OH}$ & $\alpha-\mathrm{OH}$ & $\mathrm{H}$ & $\mathrm{OCH}_{3}$ \\
\hline 69 & $\beta-\mathrm{OCH}_{3}$ & $\alpha-\mathrm{OH}$ & $\alpha-\mathrm{OH}$ & $\beta-\mathrm{OH}$ & $\mathrm{H}$ & $\mathrm{OCH}_{3}$ \\
\hline 70 & $\alpha-\mathrm{OCH}_{3}$ & $\beta-\mathrm{OH}$ & $\beta-\mathrm{OH}$ & $\alpha-\mathrm{OH}$ & $\mathrm{OH}$ & $\mathrm{OCH}_{3}$ \\
\hline 71 & $\alpha-\mathrm{OCH}_{3}$ & $\beta-\mathrm{OH}$ & $\beta-\mathrm{OH}$ & $\alpha-\mathrm{Cl}$ & $\mathrm{H}$ & $\mathrm{OCH}_{3}$ \\
\hline 72 & $\alpha-\mathrm{OH}$ & $\beta-\mathrm{OH}$ & $\beta-\mathrm{OH}$ & $\alpha-\mathrm{Cl}$ & $\mathrm{H}$ & $\mathrm{OCH}_{3}$ \\
\hline 73 & $\alpha-\mathrm{OCH}_{3}$ & $\alpha-\mathrm{OH}$ & $\alpha-\mathrm{OH}$ & $\beta-\mathrm{OH}$ & $\mathrm{H}$ & $\mathrm{OCH}_{3}$ \\
\hline 74 & $\beta-\mathrm{OCH}_{3}$ & $\beta-\mathrm{OH}$ & $\beta-\mathrm{OH}$ & $\alpha-\mathrm{OH}$ & $\mathrm{H}$ & $\mathrm{OCH}_{3}$ \\
\hline 75 & $\alpha-\mathrm{OCH}_{3}$ & $\alpha-\mathrm{OH}$ & $\alpha-\mathrm{OH}$ & $\beta-\mathrm{OH}$ & $\mathrm{OH}$ & $\mathrm{OCH}_{3}$ \\
\hline 76 & $\alpha-\mathrm{OCH}_{3}$ & $\alpha-\mathrm{OH}$ & $\alpha-\mathrm{OH}$ & $\beta-\mathrm{Cl}$ & $\mathrm{H}$ & $\mathrm{OCH}_{3}$ \\
\hline 77 & $\alpha-\mathrm{OCH}_{3}$ & $\alpha-\mathrm{OH}$ & $\alpha-\mathrm{OH}$ & $\beta-\mathrm{Cl}$ & $\mathrm{OH}$ & $\mathrm{OCH}_{3}$ \\
\hline
\end{tabular}

Figure 3. Structures of 5,6,7,8-tetrahydro-2-(2-phenylethyl)chromones identified in agarwood.<smiles>[R]c1ccc(CCc2cc(=O)c3c(o2)C([R])[C@H](O)[C@H]2O[C@@H]32)cc1[R]</smiles>

$13 \mathrm{R}^{1}=\alpha-\mathrm{OH}, \mathrm{R}^{2}=\mathrm{H}, \mathrm{R}^{3}=\mathrm{OCH}_{3}$

$14 \mathrm{R}^{1}=\alpha-\mathrm{OH}, \mathrm{R}^{2}=\mathrm{H}, \mathrm{R}^{3}=\mathrm{H}$

$15 \mathrm{R}^{1}=\alpha-\mathrm{OH}, \mathrm{R}^{2}=\mathrm{OH}, \mathrm{R}^{3}=\mathrm{OCH}_{3}$

$29 \mathrm{R}^{1}=\beta-\mathrm{OCH}_{3}, \mathrm{R}^{2}=\mathrm{H}, \mathrm{R}^{3}=\mathrm{H}$<smiles>O=c1cc(CCc2ccccc2)oc2c1[C@H](O)[C@H](O)C1OC21</smiles>

78<smiles>COc1ccc(CCc2cc(=O)c3c(o2)[C@H](O)[C@H](O)[C@H]2O[C@@H]32)cc1O</smiles>

45<smiles>[R]c1ccc(CCc2cc(=O)c3c(o2)C2O[C@H]2[C@H](O)[C@@H]3O)cc1[R]</smiles>

$79 \mathrm{R}^{1}=\mathrm{OCH}_{3}, \mathrm{R}^{2}=\mathrm{H}$

$80 \mathrm{R}^{1}=\mathrm{OCH}_{3}, \mathrm{R}^{2}=\mathrm{OH}$

Figure 4. Structures of 5,6,7,8-tetrahydro-2-(2-phenylethyl)chromones with epoxide identified in agarwood. 
<smiles>COc1ccc(CCc2cc(=O)c3c(o2)[C@H](Oc2cc4oc(CCc5ccc(O)c(OC)c5)cc(=O)c4cc2OC)[C@H](O)[C@@H](O)[C@H]3O)cc1</smiles>

30

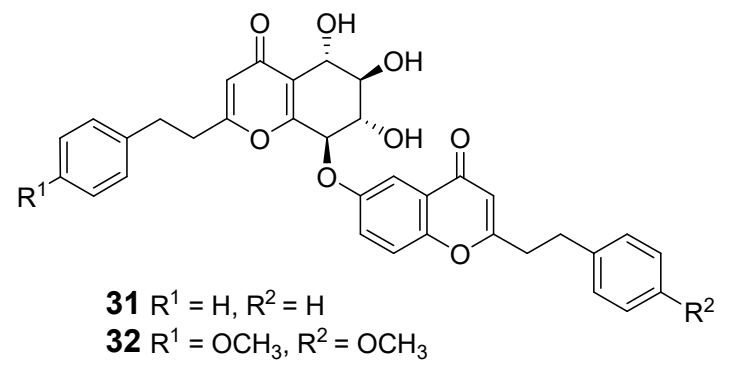<smiles>O=c1cc(CCc2ccccc2)oc2c1[C@@H](O)[C@H](O)[C@H](O)[C@H]2Oc1ccc2oc(CCc3ccccc3)cc(=O)c2c1</smiles>

$32 \mathrm{R}^{1}=\mathrm{OCH}_{3}, \mathrm{R}^{2}=\mathrm{OCH}_{3}$

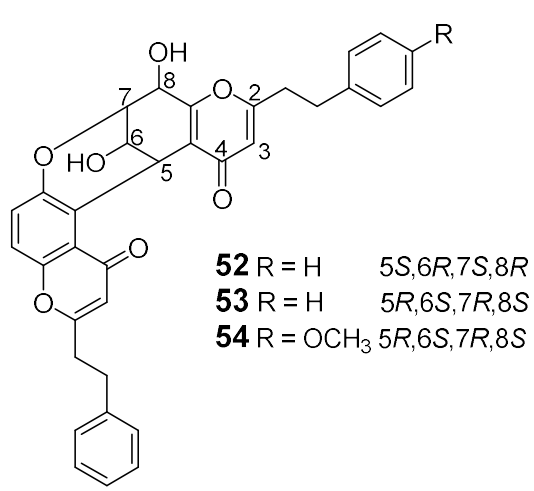

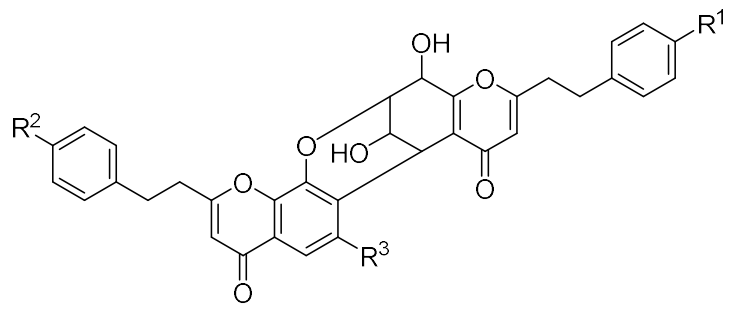

$55 \mathrm{R}^{1}=\mathrm{R}^{2}=\mathrm{R}^{3}=\mathrm{H} \quad 5 R, 6 \mathrm{~S}, 7 R, 8 \mathrm{~S}$

$56 \mathrm{R}^{1}=\mathrm{R}^{2}=\mathrm{R}^{3}=\mathrm{H} \quad 5 \mathrm{~S}, 6 \mathrm{R}, 7 \mathrm{~S}, 8 \mathrm{R}$

$57 \mathrm{R}^{1}=\mathrm{R}^{2}=\mathrm{H}, \mathrm{R}^{3}=\mathrm{OH} \quad 5 \mathrm{~S}, 6 \mathrm{R}, 7 \mathrm{~S}, 8 \mathrm{R}$

$58 \mathrm{R}^{1}=\mathrm{R}^{2}=\mathrm{OCH}_{3}, \mathrm{R}^{3}=\mathrm{OH} 5 R, 6 \mathrm{~S}, 7 R, 8 \mathrm{~S}$

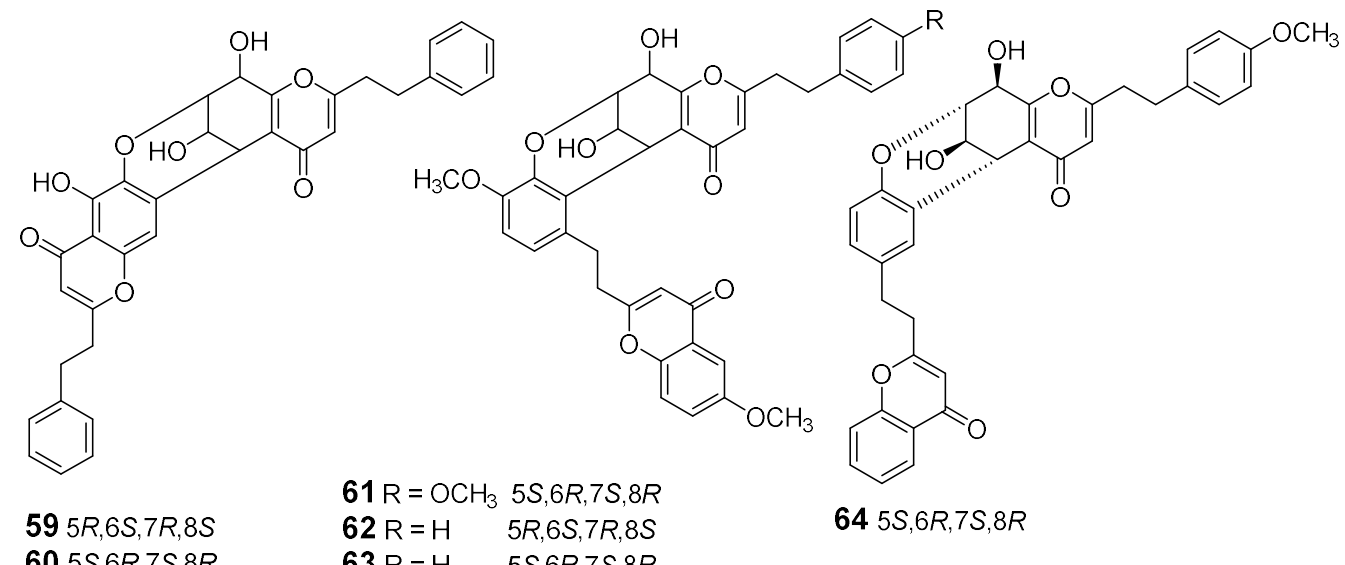

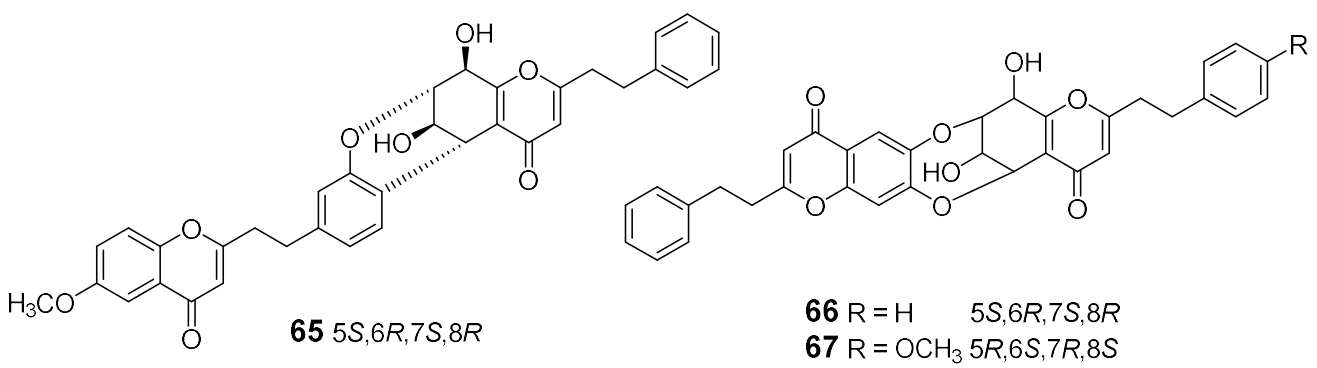

Figure 5. Structures of dimeric 2-(2-phenylethyl)chromones identified in agarwood. 


\subsubsection{Terpenoids}

Terpenoids are compounds derived from mevalonic acid, whose basic carbon frame is characterized by having two or more isoprene units. Terpenoids, including sesquiterpenes and diterpenes, are the main components of agarwood. The EtOH extract of agarwood was isolated and, as a result, a total of 34 new sesquiterpenes (89-117, 131-135) (Figure 6) were gained [30-36], in which nine compounds (102-104, 110-115) were identified from "Qi-Nan" [32,34]. The isolation of a petroleum ether fraction obtained two new sesquiterpene derivatives $(\mathbf{1 1 8}, \mathbf{1 1 9})$ (Figure 6) [37], and eleven new diterpenoids (120-130) (Figure 7) were identified from EtOH extract [38]. Additionally, many new terpenoids have also been found in other parts of genus Aquilaria plants. Peng et al. [39] isolated a novel degraded sesquiterpene, named aquilarin B (131) (Figure 6) from the EtOH extract of the fresh stem (A. sinensis) and Cheng et al. [40] got two new tirucallane triterpenoids (136-137) (Figure 8) from the leaves of $A$. sinensis. Furthermore, aquimavitalin (138) and four new phorbol esters (139-142) were isolated from an A. malaccensis seeds ethanolic extract [41,42] (Figure 9).<smiles>[R]C(=C)C1CC2=C(C)CCCC2(C)C([R])C1Br</smiles>

$89 \mathrm{R}^{1}=\mathrm{OH}, \mathrm{R}^{2}=\mathrm{H}, \mathrm{R}^{3}=\mathrm{CHO}$ $90 \mathrm{R}^{1}=\mathrm{OH}, \mathrm{R}^{2}=\mathrm{OH}, \mathrm{R}^{3}=\mathrm{CH}_{3}$<smiles>C=C(C)C1CC(O)C2(C)CCCC(C)(C)C1C2</smiles>

94

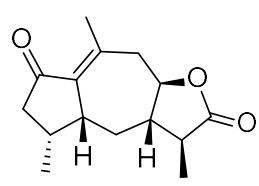

98<smiles>C=C(C)C1CCC(C)=C2CCC(C)C2C1</smiles>

102<smiles>C=C(C)C1CC(O)C2(C)CCC=C(C=O)C2C1</smiles>

106<smiles>C=C(C)C1CC2OC23CCCC(C)C13C</smiles>

95<smiles>CC(=O)CC1OC(=O)C(C)C1CC1C(=O)C=CC1C</smiles>

99<smiles>C=C(C)C1CCC2(C)CCC=C(O)C2C1</smiles>

103<smiles>C=C(C(=O)OC)C1CC2C(C)=CCCC2(C)[C@@H](O)C1</smiles><smiles>[R]C1=CCCC2(C)C1CC(C(=C)C)C(O)C2[R]</smiles>

$91 \mathrm{R}^{1}=\mathrm{OH}, \mathrm{R}^{2}=\mathrm{H}, \mathrm{R}^{3}=\mathrm{CHO}$ $92 \mathrm{R}^{1}=\mathrm{OH}, \mathrm{R}^{2}=\mathrm{OH}, \mathrm{R}^{3}=\mathrm{CH}_{3}$<smiles>C[C@H]1CC(=O)C=C2C=CC(C(C)(C)O)C[C@H]21</smiles>

96

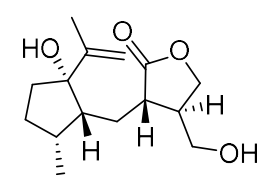

100<smiles>C=C(C)C1CC23CCCCC2(C)C(C)CC3=CC1=O</smiles>

104<smiles>C=C(C)C1CC(O)[C@@]2(C)CC1CC1(C)CCCC(C=O)=C12</smiles>

108<smiles>C[C@@H]1CC[C@H]2CC(=O)C[C@@H](O)[C@@H]2C1</smiles>

101<smiles>C=C1CCCC2(C)C1C[C@@H](C(=C)C)C(O)C2O</smiles>

93<smiles>CC1CCCC2=CC(O)C(C(C)(C)O)C[C@H]2C1</smiles>

97<smiles>C=C(C=O)C1CC(O)C2(C)CCC=C(C)C2C1</smiles>

105<smiles>C=C(CO)C1CC2=C(C)CCCC2(C)C[C@H]1O</smiles>

109

Figure 6. Cont. 


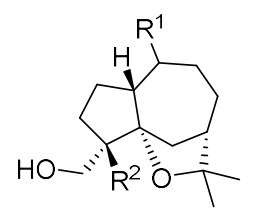

$110 \mathrm{R}^{1}=\alpha-\mathrm{CH}_{3}, \mathrm{R}^{2}=\mathrm{H}$

$111 R^{1}=\beta-\mathrm{CH}_{3}, \mathrm{R}^{2}=\mathrm{H}$

$112 \mathrm{R}^{1}=\alpha-\mathrm{CH}_{3}, \mathrm{R}^{2}=\mathrm{OH}$

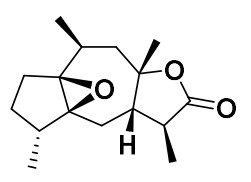

117

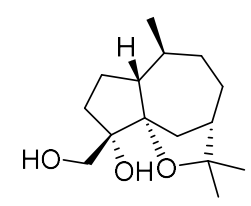

113

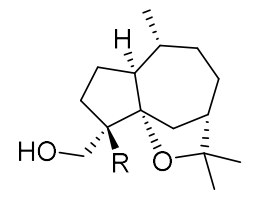

$114 \mathrm{R}=\mathrm{OH}$

$115 \mathrm{R}=\mathrm{H}$

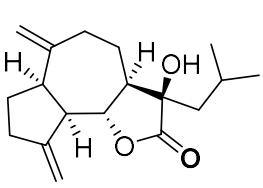

119

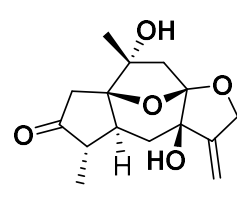

116

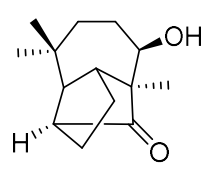

118

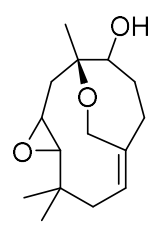

$( \pm) 133$

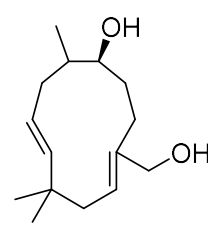

134

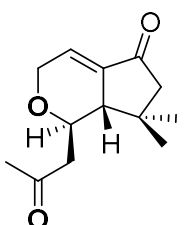

131

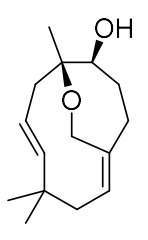

132

Figure 6. Structures of sesquiterpenes identified in agarwood.

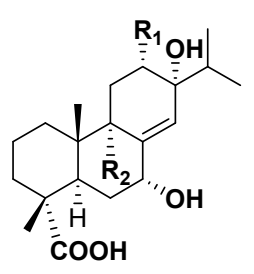

$120 \mathrm{R}^{1}=\mathrm{H}, \mathrm{R}^{2}=\mathrm{OH}$

$121 \mathrm{R}^{1}=\mathrm{OH}, \mathrm{R}^{2}=\mathrm{H}$<smiles>CC(C)C12CCC3C(=C[C@@H]4OC(=O)[C@]5(C)CCC[C@]4(C)[C@H]35)C1O2</smiles>

125<smiles>CCO[C@H]1CC2C(C=C1C(C)C)CC[C@H]1C(C)(C)CCC[C@@]21C</smiles>

129<smiles>CC(C)O[C@@H]1C[C@@H](O)CC2C1=C[C@@](OC(C)(C)C)(C(C)C)C[C@H]1[C@@H](C)CCC[C@]21C</smiles>

122<smiles>CC(C)[C@]1(O)C=C2C(C[C@H](O)C1)[C@@]1(C)CCC[C@](C)(C(=O)O)[C@H]1C[C@H]2O</smiles>

126

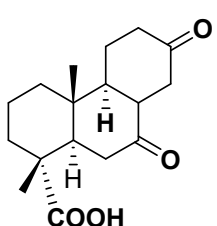

130<smiles>CC(C)[C@]1(O)CC[C@H]2C(=C[C@H](O)C3[C@@H](C)CCCC[C@]32C)[C@@H]1O</smiles>

123<smiles>C=C(C)c1ccc2c(c1)[C@H](O)C[C@H]1[C@@](C)(C(=O)O)CCC[C@]21C</smiles>

127

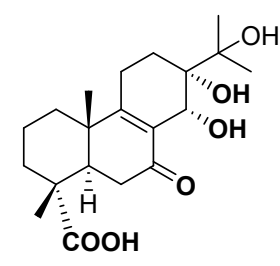

124

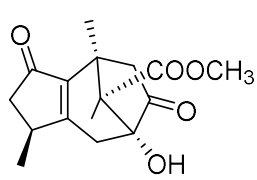

135 
<smiles>C=C(CCC(C)[C@H]1CCC2(C)C3=CC[C@H]4[C@@H](C)[C@H](O)CC[C@]4(C)[C@H]3CC[C@]12C)C(C)(C)O</smiles>

136<smiles>C=C(CCC(C)[C@H]1CC[C@]2(C)C3=C(C(=O)C[C@H]12)C1(C)CC[C@@H](O)[C@H](C)C1C3=O)C(C)(C)C</smiles>

137

Figure 8. Structures of tirucallane triterpenoids from Aquilaria sinensis.

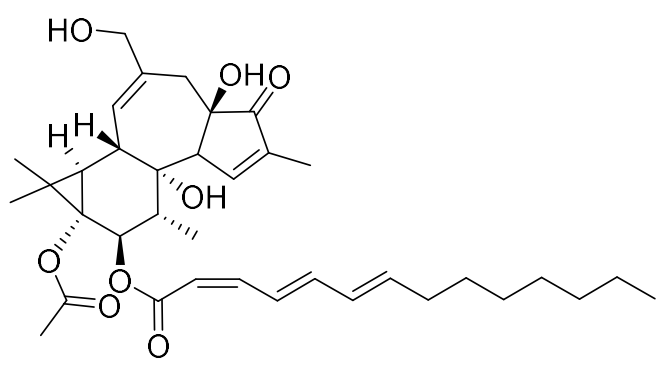

138

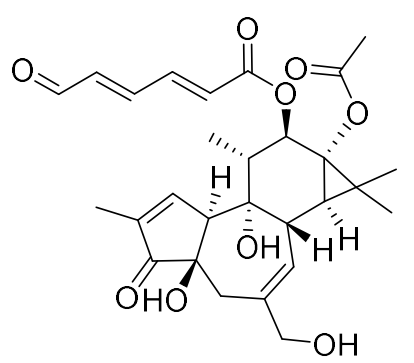

139

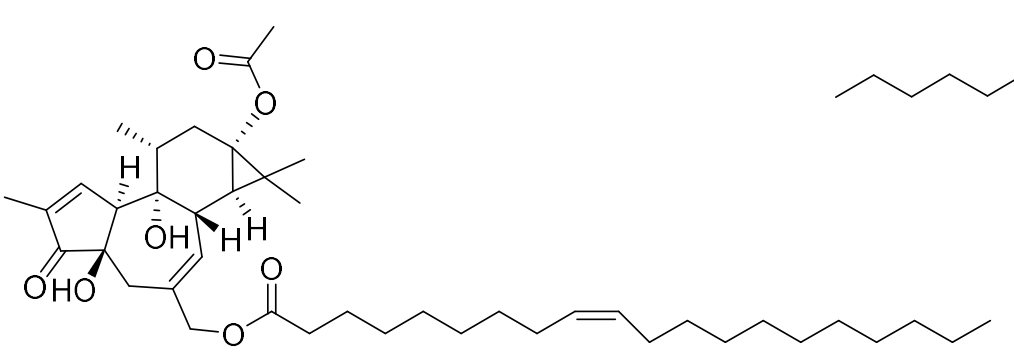

140
141 erythro

142 threo

Figure 9. Structures of phorbol esters from Aquilaria malaccensis.

\subsubsection{Flavonoids}

Flavonoids consist of a large group of polyphenolic compounds with a benzo- $\gamma$-pyrone structure, which is ubiquitously present in plants; there is no exception for the genus Aquilaria plants. Two new flavones $(143,144)$ were obtained from the EtOAc fraction of stem bark (A. sinensis) [27] (Figure 10). Another six new flavonoids (145-150) were isolated from the leaves of A. sinensis [43-45] (Figure 10). 
<smiles>COc1cc(O)c2c(=O)cc(-c3cc(OC)c(OC/C=C(\C)CCC=C(C)C)c(OC)c3)oc2c1</smiles><smiles>COc1cc(OCC2OC(C(O)O)C(O)C(O)C(O)C(O)C(O)C2O)c2c(=O)cc(-c3ccc(O)c(OC)c3)oc2c1</smiles>

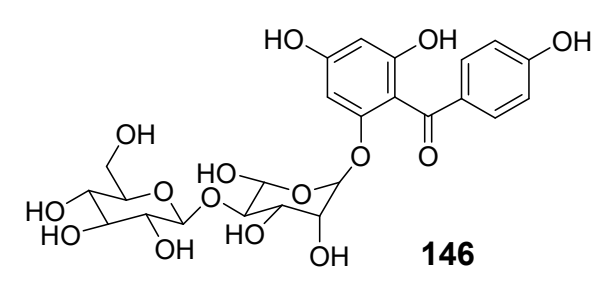

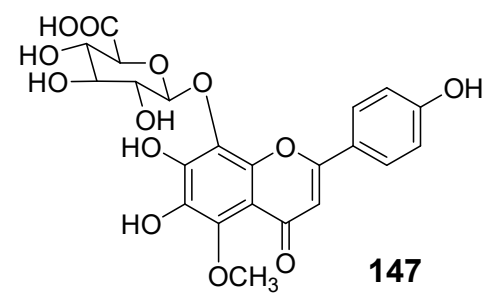

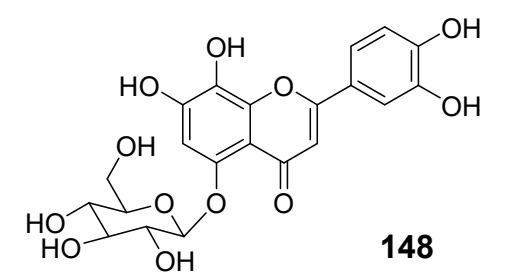<smiles>O=c1c2cc(O)c(O)cc2oc2c(O)c(O)c(C3OC(O)C4COC3C(O)O4)c(O)c12</smiles><smiles></smiles>

Figure 10. Structures of flavonoids from Aquilaria malaccensis.

\subsubsection{Others}

Compounds 151-154 are included here, as they do not belong to any of the above classes [45-47] (Figure 11).<smiles>O=C(c1ccc(O)cc1)c1c(O)cc(O)c2c1OC1(C2)OC(CO)C(O)C1O</smiles>

151<smiles>COc1cc(C(=O)[C@H]2CCC(=O)[C@H]2CO)cc(OC)c1O</smiles>

152<smiles>CC1=C[C@@H](O)C(O)C(C)(C)[C@H]1/C=C/[C@H](C)OC(O)C(O)C(O)C1OC(O)C(O)C1O</smiles>

153<smiles>C=C1C[C@H](O)CC(C)(C)[C@]1(O)/C=C/[C@H](C)OC(O)C(O)(O)C(O)(O)C(O)O</smiles>

\section{4}

Figure 11. Structures of compounds from agarwood. 
Table 1. Chemical constituents of agarwood originating from the genus Aquilaria.

\begin{tabular}{|c|c|c|c|c|}
\hline No. & Compound Class and Name & Source or Origin & Extraction* & Ref. \\
\hline \multicolumn{5}{|c|}{ 2-(2-Phenylethyl)chromones } \\
\hline 1 & 7-Hydroxy-6-methoxy-2-[2-(3'-hydroxy-4'-ethoxyphenyl)ethyl]chromone & A. sinensis (China) & EtOH & [16] \\
\hline 2 & 6,7-Dimethoxy-2-[2-(4'-hydroxy-3'-methoxyphenyl) ethyl]chromone & A. sinensis (China) & $\mathrm{EtOH}$ & [16] \\
\hline 3 & 6,7-Dihydroxy-2-[2-(4'-methoxyphenyl)ethyl]chromone & A. sinensis (China) & EtOH & [16] \\
\hline 4 & 6-Hydroxy-7-methoxy-2-[2-(4'-hydroxyphenyl)ethyl]chromone & A. sinensis (China) & EtOH & [16] \\
\hline 5 & 6,8-Dihydroxy-2-[2-(3'-hydroxy4'-methoxyphenyl)ethyl]chromone & A. sinensis (China) & EtOH & [16] \\
\hline 6 & 6-Hydroxy-2-[2-(4'-hydroxy-3'-methoxyphenyl)ethenyl]chromone & A. sinensis (China) & $\mathrm{EtOH}$ & [16] \\
\hline 7 & 6-Hydroxy-7-methoxy2-[2-(3'-hydroxy-4'-methoxyphenyl)ethyl]chromone & A. sinensis (China) & EtOH & [16] \\
\hline 8 & 6,7-Dimethoxy-2-[2-(3'-hydroxy-4'-xyphenyl)ethyl]chromone & A. sinensis (China) & EtOH & [16] \\
\hline 9 & 5,6,7,8-Tetrahydroxy-2-(3-hydroxy-4-methoxyphenethyl)-5,6,7,8-tetrahydro-4H-chromen-4-one & A. sinensis (China) & $\mathrm{EtOH}-\mathrm{H}_{2} \mathrm{O}$ & [17] \\
\hline 10 & 8-Chloro-6-hydroxy-2-(2-phenylethyl)chromen-4-one & A. sinensis (China) & EtOH-EtOAc & [18] \\
\hline 11 & 8-Chloro-6-hydroxy-2-[2-(4-methoxyphenyl)ethyl]chromen-4-one & A. sinensis (China) & EtOH-EtOAc & [18] \\
\hline 12 & Rel-(5R,6S,7R)-5,6,7,8-Tetrahydro-5,6,7-trihydroxy-2-(2-phenylethyl)-4H-1-benzopyran-4-one & A. malaccensis (Laos) & EtOH- $n-\mathrm{BuOH}$ & [19] \\
\hline 13 & Rel-(5R,6S,7R)-5,6,7,8-Tetrahydro-5,6,7-trihydroxy-2-[2-(4-methoxyphenyl)ethyl]-4H-1-benzopyran4-one & A. malaccensis (Laos) & EtOH- $n$-BuOH & [19] \\
\hline 14 & 7-Hydroxy-6-methoxy-2-[2-(4-methoxyphenyl)ethyl]-4H-1-benzopyran-4-one & A. malaccensis (Laos) & EtOH- $n$-BuOH & [19] \\
\hline 15 & Rel-(1aR,2R,3R,7bS)-1 $\alpha, 2,3,7 \beta$-Tetrahydro-2,3-dihydroxy-5-[2-(4-methoxyphenyl)ethyl]-7H-oxireno[f] [1]benzopyran-7-one & A. malaccensis (Laos) & EtOH-n-BuOH & [19] \\
\hline 16 & Rel-(1aR,2R,3R,7bS)-1 $\alpha, 2,3,7 \beta$-Tetrahydro-2,3-dihydroxy-5-(2-phenylethyl)-7H-oxireno[f] [1]benzopyran-7-one & A. malaccensis (Laos) & EtOH- $n$-BuOH & [19] \\
\hline 17 & Rel-(1aR,2R,3R,7bS)-1 $\alpha, 2,3,7 \beta$-Tetrahydro-2,3-dihydroxy-5-[2-(3-hydroxy-4-methoxyphenyl)ethyl]-7H-oxireno[f] [1]benzopyran-7-one & A. malaccensis (Laos) & EtOH- $n$-BuOH & [19] \\
\hline 18 & Rel-(5R,6S,7SS,8R)-8-Chloro-5,6,7,8-tetrahydro-5,6,7-trihydroxy-2-[2-(4-methoxyphenyl)ethyl]-4H-1-benzopyran-4-one & A. malaccensis (Laos) & EtOH-n-BuOH & [19] \\
\hline 19 & Rel-(5R,6S,7S, BR)-8-Chloro-5,6,7,8-tetrahydro-5,6,7-trihydroxy-2-[2-(3-hydroxy-4-methoxyphenyl)ethyl]-4H-1-benzopyran-4-one & A. malaccensis (Laos) & EtOH- $n$-BuOH & [19] \\
\hline 20 & 6-Hydroxy-7-methoxy-2-[2-(4-methoxyphenyl)ethyl]chromone & A. sinensis (China) & EtOH-EtOAc & [20] \\
\hline 21 & 6-Hydroxy-2-[2-(3,4-dimethoxyphenyl)ethyl]chromone & A. sinensis (China) & EtOH-EtOAc & [20] \\
\hline 22 & 6,8-Dihydroxy-2-[2-(4-methoxyphenyl)ethyl]chromone & A. sinensis (China) & EtOH-EtOAc & [20] \\
\hline 23 & 8-Chloro-6-hydroxy-2-[2-(3-methoxy-4-hydroxyphenyl)ethyl]chromone & A. sinensis (China) & EtOH-EtOAc & [20] \\
\hline 24 & 5-Methoxy-6-hydroxy-2-[2-(3-methoxy-4-hydroxyphenyl)ethyl]chromone & A. sinensis (China) & EtOH-EtOAc & [20] \\
\hline 25 & (R)-6,7-Dimethoxy-2-(2-hydroxy-2-phenylethyl)chromone & A. sinensis (China) & EtOH-EtOAc & [20] \\
\hline 26 & (S)-6,7-Dimethoxy-2-(2-hydroxy-2-phenylethyl)chromone & A. sinensis (China) & EtOH-EtOAc & [20] \\
\hline 27 & 6-Methoxy-2-[2-(3-hydroxy-4-methoxyphenyl)ethyl]chromone & A. sinensis (China) & EtOH-EtOAc & [8] \\
\hline 28 & 5-Hydroxy-6-methoxy-2-[2-(3-hydroxy-4-methoxyphenyl)ethyl]chromone & A. sinensis (China) & EtOH-EtOAc & [8] \\
\hline 29 & 5,6-Epoxy-7 $\beta$-hydroxy-8 $\beta$-methoxy-2-(2-phenylethyl)chromone & A. sinensis (China) & EtOH-EtOAc & [8] \\
\hline 30 & $\begin{array}{l}(5 S, 6 R, 7 S, 8 R)-2-[2-(4-M e t h o x y p h e n y l) \text { ethyl]-5,6,7-trihydroxy-5,6,7,8-tetrahydro-8-\{6-methoxy-2- } \\
{\left[2-\left(3^{\prime \prime \prime}-\text {-methoxy-4"' }\right.\right.}\end{array}$ & A. sinensis (China) & EtOH-EtOAc & [21] \\
\hline 31 & (5S,6R,7S,8R)-2-[2-(4-Methoxyphenyl)ethyl]-5,6,7-trihydroxy-5,6,7,8-Tetrahydro-8-\{2-[2-(4'"'-methoxyphenyl)ethyl]chromonyl-6-oxy\}chromone & A. sinensis (China) & EtOH-EtOAc & [21] \\
\hline 32 & (5S,6R,7S, 8R)-2-(2-Phenylethyl)-5,6,7-trihydroxy-5,6,7,8-tetrahydro-8-[2-(2-phenylethyl)chromonyl-6-oxy]chromone & A. sinensis (China) & EtOH-EtOAc & [21] \\
\hline 33 & $(5 R, 6 R, 7 R, 8 S)$-2-(2-Phenylethyl)-5,6,7-trihydroxy-5,6,7,8-tetrahydro-8-[2-(2-phenylethyl)chromonyl-6-oxy]chromone & A. sinensis (China) & EtOH-EtOAc & [21] \\
\hline 34 & Aquilarone A & A. sinensis (China) & $\mathrm{EtOH}-\mathrm{CHCl}_{3}$ & [22] \\
\hline 35 & Aquilarone B & A. sinensis (China) & $\mathrm{EtOH}-\mathrm{CHCl}_{3}$ & [22] \\
\hline 36 & Aquilarone $\mathrm{C}$ & A. sinensis (China) & $\mathrm{EtOH}-\mathrm{CHCl}_{3}$ & [22] \\
\hline 37 & Aquilarone D & A. sinensis (China) & $\mathrm{EtOH}-\mathrm{CHCl}_{3}$ & [22] \\
\hline 38 & Aquilarone $\mathrm{E}$ & A. sinensis (China) & $\mathrm{EtOH}-\mathrm{CHCl}_{3}$ & [22] \\
\hline 39 & Aquilarone $\mathrm{F}$ & A. sinensis (China) & $\mathrm{EtOH}-\mathrm{CHCl}_{3}$ & [22] \\
\hline 40 & Aquilarone G & A. sinensis (China) & $\mathrm{EtOH}-\mathrm{CHCl}_{3}$ & [22] \\
\hline 41 & Aquilarone $\mathrm{H}$ & A. sinensis (China) & $\mathrm{EtOH}-\mathrm{CHCl}_{3}$ & [22] \\
\hline 42 & Aquilarone I & A. sinensis (China) & $\mathrm{EtOH}-\mathrm{CHCl}_{3}$ & [22] \\
\hline 43 & 5-Hydroxy-7-methoxy-2-[2-(4-methoxyphenyl)ethyl]chromone & A. sinensis (China) & $\mathrm{EtOH}-\mathrm{CH}_{2} \mathrm{Cl}_{2}$ & [23] \\
\hline
\end{tabular}


Table 1. Cont

\begin{tabular}{|c|c|c|c|c|}
\hline No. & Compound Class and Name & Source or Origin & Extraction * & Ref. \\
\hline \multicolumn{5}{|c|}{ 2-(2-Phenylethyl)chromones } \\
\hline 44 & 5,8-Dihydroxy-6-methoxy-2-(2-phenylethyl)chromone & A. sinensis (China) & $\mathrm{EtOH}-\mathrm{CH}_{2} \mathrm{Cl}_{2}$ & [23] \\
\hline 45 & $5 \alpha, 6 \alpha$-Epoxy-7 $\beta, 8 \alpha, 30$-trihydroxy-40-methoxy-2-(2-phenylethyl)chromone & A. sinensis (China) & $\mathrm{EtOH}-\mathrm{CH}_{2} \mathrm{Cl}_{2}$ & [23] \\
\hline 46 & 6-Methoxy-2-[2-(20,30,40-trihydroxy)phenyl)ethyl]chromone & A. sinensis (China) & $\mathrm{EtOH}-\mathrm{CH}_{2} \mathrm{Cl}_{2}$ & [23] \\
\hline 47 & 5-Hydroxy-6,7-dimethoxy-2-[2-(4'-methoxyphenyl)ethyl]chromone & A. sinensis (China) & EtOH-EtOAc & [24] \\
\hline 48 & (5R,6R,7R,8S)-8-Chloro-5,6,7-trihydroxy-2-(4-methoxyphenethyl)-5,6,7,8-tetrahydrochromone & A. sinensis (China) & EtOH-EtOAc & [24] \\
\hline 49 & (5S,6S,7S,8S)-8-Chloro-5,6,7-trihydroxy-2-(2-phenylethyl)-5,6,7,8-tetrahydrochromone & A. sinensis (China) & EtOH-EtOAc & [24] \\
\hline 50 & (5R,6R,7R,8R)-8-Chloro-5,6,7-trihydroxy-2-(4-methoxyphenethyl)-5,6,7,8-tetrahydrochromone & A. sinensis (China) & EtOH-EtOAc & [24] \\
\hline 51 & $(5 R, 6 S, 7 S)-5,6,7$-Trihydroxy-2-(4-hydroxy-3-methoxyphenethyl)-5,6,7,8-tetrahydrochromone & A. sinensis (China) & EtOH-EtOAc & [24] \\
\hline 52 & $(5 S, 6 R, 7 S, 8 R)$ Aquisinenone A & A. sinensis (China) & EtOH-EtOAc & [25] \\
\hline 53 & $(5 R, 6 S, 7 R, 8 S)$ Aquisinenone $\mathrm{A}$ & A. sinensis (China) & EtOH-EtOAc & [25] \\
\hline 54 & $(-)-4^{\prime}$-Methoxyaquisinenone A & A. sinensis (China) & EtOH-EtOAc & [25] \\
\hline 55 & $(5 R, 6 S, 7 R, 8 S)$ Aquisinenone $B$ & A. sinensis (China) & EtOH-EtOAc & [25] \\
\hline 56 & $(5 S, 6 R, 7 S, 8 R)$ Aquisinenone $B$ & A. sinensis (China) & EtOH-EtOAc & [25] \\
\hline 57 & $(-)-6^{\prime \prime}-$ Hydroxyaquisinenone $\mathrm{B}$ & A. sinensis (China) & EtOH-EtOAc & [25] \\
\hline 58 & (+)-6"-Hydroxy-4', $4^{\prime \prime \prime}$-dimethoxyaquisinenone B & A. sinensis (China) & EtOH-EtOAc & [25] \\
\hline 59 & $(5 R, 6 S, 7 R, 8 S)$ Aquisinenone $\mathrm{C}$ & A. sinensis (China) & EtOH-EtOAc & [25] \\
\hline 60 & $(5 S, 6 R, 7 S, 8 R)$ Aquisinenone $C$ & A. sinensis (China) & EtOH-EtOAc & [25] \\
\hline 61 & (-)-Aquisinenone D & A. sinensis (China) & EtOH-EtOAc & [25] \\
\hline 62 & $(5 R, 6 S, 7 R, 8 S) 4^{\prime}$-Demethoxyaquisinenone D & A. sinensis (China) & EtOH-EtOAc & [25] \\
\hline 63 & $(5 S, 6 R, 7 S, 8 R) 4^{\prime}$-Demethoxyaquisinenone D & A. sinensis (China) & EtOH-EtOAc & [25] \\
\hline 64 & (+)-Aquisinenone $\mathrm{E}$ & A. sinensis (China) & EtOH-EtOAc & [25] \\
\hline 65 & $(-)$-Aquisinenone $\mathrm{F}$ & A. sinensis (China) & EtOH-EtOAc & [25] \\
\hline 66 & (-)-Aquisinenone G & A. sinensis (China) & EtOH-EtOAc & [25] \\
\hline 67 & (+)-4'-Methoxyaquisinenone $\mathrm{G}$ & A. sinensis (China) & EtOH-EtOAc & [25] \\
\hline 68 & Tetrahydrochromone $\mathrm{A}$ & A. sinensis (China) & EtOH-EtOAc & [26] \\
\hline 69 & Tetrahydrochromone B & A. sinensis (China) & EtOH-EtOAc & [26] \\
\hline 70 & Tetrahydrochromone $\mathrm{C}$ & A. sinensis (China) & EtOH-EtOAc & [26] \\
\hline 71 & Tetrahydrochromone D & A. sinensis (China) & EtOH-EtOAc & [26] \\
\hline 72 & Tetrahydrochromone E & A. sinensis (China) & EtOH-EtOAc & [26] \\
\hline 73 & Tetrahydrochromone $\mathrm{F}$ & A. sinensis (China) & EtOH-EtOAc & [26] \\
\hline 74 & Tetrahydrochromone G & A. sinensis (China) & EtOH-EtOAc & [26] \\
\hline 75 & Tetrahydrochromone $\mathrm{H}$ & A. sinensis (China) & EtOH-EtOAc & [26] \\
\hline 76 & Tetrahydrochromone I & A. sinensis (China) & EtOH-EtOAc & [26] \\
\hline 77 & Tetrahydrochromone J & A. sinensis (China) & EtOH-EtOAc & [26] \\
\hline 78 & Tetrahydrochromone $\mathrm{K}$ & A. sinensis (China) & EtOH-EtOAc & [26] \\
\hline 79 & Tetrahydrochromone L & A. sinensis (China) & EtOH-EtOAc & [26] \\
\hline 80 & Tetrahydrochromone $\mathrm{M}$ & A. sinensis (China) & EtOH-EtOAc & [26] \\
\hline 81 & 7-Hydroxyl-6-methoxy-2-(2-phenylethyl)chromone & A. sinensis (China) & EtOH-EtOAc & [27] \\
\hline 82 & Qinanone A & A. sinensis (China) & $\mathrm{EtOH}-\mathrm{Et}_{2} \mathrm{O}$ & [28] \\
\hline 83 & Qinanone B & A. sinensis (China) & $\mathrm{EtOH}-\mathrm{Et}_{2} \mathrm{O}$ & [28] \\
\hline 84 & Qinanone C & A. sinensis (China) & $\mathrm{EtOH}-\mathrm{Et}_{2} \mathrm{O}$ & [28] \\
\hline 85 & Qinanone D & A. sinensis (China) & $\mathrm{EtOH}-\mathrm{Et}_{2} \mathrm{O}$ & [28] \\
\hline 86 & Qinanone E & A. sinensis (China) & $\mathrm{EtOH}-\mathrm{Et}_{2} \mathrm{O}$ & [28] \\
\hline 87 & Qinanone $\mathrm{G}$ & A.sinensis (China) & $\mathrm{EtOH}-\mathrm{Et}_{2} \mathrm{O}$ & [28] \\
\hline 88 & 2-(2-Hydroxy-2-phenylethyl)-4H-chromen-4-one & A. filaria (Japan) & $\mathrm{EtOH}-\mathrm{MeOH}$ & [29] \\
\hline
\end{tabular}


Table 1. Cont

\begin{tabular}{|c|c|c|c|c|}
\hline No. & Compound Class and Name & Source or Origin & Extraction * & Ref. \\
\hline \multicolumn{5}{|c|}{ Terpenoids } \\
\hline 89 & (+)-9 9 -Hydroxyeudesma-4,11(13)-dien-12-al & A.sinensis (China) & EtOH-petroleum ether & [30] \\
\hline 90 & (+)-Eudesma-4,11(13)-dien-8 $\alpha, 9 \beta$-diol & A.sinensis (China) & EtOH-petroleum ether & [30] \\
\hline 91 & (+)-8 8 -Hydroxyeudesma-3,11(13)-dien-14-al & A.sinensis (China) & EtOH-petroleum ether & [30] \\
\hline 92 & (+)-Eudesma-3,11(13)-dien-8 $\alpha \alpha, 9 \beta$-diol & A.sinensis (China) & EtOH-petroleum ether & [30] \\
\hline 93 & 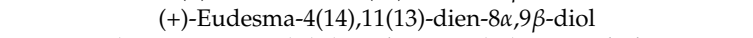 & A.sinensis (China) & EtOH-petroleum ether & [30] \\
\hline 94 & $(4 R, 5 R, 7 S, 9 S, 10 S)$-(-)-Eudesma-11(13)-en-4,9-diol & A.sinensis (China) & EtOH-petroleum ether & [30] \\
\hline 95 & $(+)-9 \beta, 10 \beta$-Epoxyeremophila-11(13)-en & A.sinensis (China) & EtOH-petroleum ether & [30] \\
\hline 96 & (+)-11-Hydroxyvalenc-1(10),8-dien-2-one & A.sinensis (China) & EtOH-petroleum ether & [30] \\
\hline 97 & (-)-Eremophila-9-en-8 $\beta, 11$-diol & A.sinensis (China) & EtOH-petroleum ether & [30] \\
\hline 98 & 1,10-Dioxo-4H-5H-7H-11H-1,10-secoguaia-2(3)-en-12,8-olide & A. sinensis (China) & EtOH & [31] \\
\hline 99 & 1-Hydroxy-4H-5H-7H-11H-8,9-secoguaia-9(10)-en-8,12-olide & A. sinensis (China) & $\mathrm{EtOH}$ & [31] \\
\hline 100 & 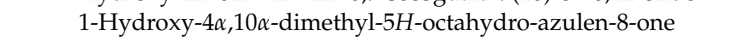 & A. sinensis (China) & EtOH & [31] \\
\hline 101 & $1 \alpha$-Hydroxy- $4 \alpha, 10 \alpha$-dimethyl- $5 \beta H$-octahydro-azulen-8-one & A. sinensis (China) & $\mathrm{EtOH}$ & [31] \\
\hline 102 & 4-Hydroxyl-baimuxinol & A. sinensis (China) & $\mathrm{EtOH}-\mathrm{Et}_{2} \mathrm{O}$ & [32] \\
\hline 103 & 7ß-H-9(10)-ene-11,12-Epoxy-8-oxoeremophilane & A. sinensis (China) & $\mathrm{EtOH}-\mathrm{Et}_{2} \mathrm{O}$ & [32] \\
\hline 104 & $7 \alpha$-H-9(10)-ene-11,12-Epoxy-8-oxoeremophilane & A. sinensis (China) & $\mathrm{EtOH}-\mathrm{Et}_{2} \mathrm{O}$ & [32] \\
\hline 105 & $(5 S, 7 S, 9 S, 10 S)-(+)-9$-Hydroxy-selina-3,11-dien-12-al & A. sinensis (China) & EtOH-EtOAc & [33] \\
\hline 106 & $(5 S, 7 S, 9 S, 10 S)-(-)-9-H y d r o x y-s e l i n a-3,11-d i e n-14-a l$ & A. sinensis (China) & EtOH-EtOAc & [33] \\
\hline 107 & $(5 S, 7 S, 9 S, 10 S)-(+)-9$-Hydroxy-eudesma-3,11(13)-dien-12-methyl ester & A. sinensis (China) & EtOH-EtOAc & [33] \\
\hline 108 & $(7 S, 9 S, 10 S)-(+)-9-H y d r o x y-s e l i n a-4,11-d i e n-14-a l$ & A. sinensis (China) & EtOH-EtOAc & [33] \\
\hline 109 & $(7 S, 8 S, 10 S)-(+)-8,12$-Dihydroxy-selina-4,11-dien-14-al & A. sinensis (China) & EtOH-EtOAc & [33] \\
\hline 110 & Qinanol A & A. sinensis (China) & $\mathrm{EtOH}-\mathrm{Et}_{2} \mathrm{O}$ & [34] \\
\hline 111 & Qinanol B & A. sinensis (China) & $\mathrm{EtOH}-\mathrm{Et}_{2} \mathrm{O}$ & [34] \\
\hline 112 & Qinanol C & A. sinensis (China) & $\mathrm{EtOH}-\mathrm{Et}_{2} \mathrm{O}$ & [34] \\
\hline 113 & Qinanol D & A. sinensis (China) & $\mathrm{EtOH}-\mathrm{Et}_{2} \mathrm{O}$ & [34] \\
\hline 114 & Qinanol E & A. sinensis (China) & $\mathrm{EtOH}-\mathrm{Et}_{2} \mathrm{O}$ & [34] \\
\hline 115 & Qinanol F & A. sinensis (China) & $\mathrm{EtOH}-\mathrm{Et}_{2} \mathrm{O}$ & [34] \\
\hline 116 & 3-oxo-7-Hydroxylholosericin A & A. sinensis (China) & EtOH-EtOAc & [35] \\
\hline 117 & 1,5,8,12-Diepoxy-guaia-12-one & A. sinensis (China) & EtOH-EtOAc & [35] \\
\hline 118 & 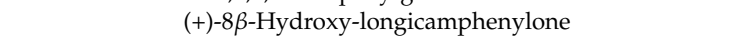 & A. sinensis (China) & EtOH-petroleum ether & [37] \\
\hline 119 & $11 \beta$-Hydroxy-13-isopropyl-dihydrodehydrocostus lactone & A. sinensis (China) & EtOH-petroleum ether & [37] \\
\hline 120 & Aquilarabietic acid A & A. sinensis (China) & EtOH & [38] \\
\hline 121 & Aquilarabietic acid B & A. sinensis (China) & $\mathrm{EtOH}$ & [38] \\
\hline 122 & Aquilarabietic acid C & A. sinensis (China) & EtOH & [38] \\
\hline 123 & Aquilarabietic acid D & A. sinensis (China) & $\mathrm{EtOH}$ & [38] \\
\hline 124 & Aquilarabietic acid E & A. sinensis (China) & EtOH & [38] \\
\hline 125 & Aquilarabietic acid $\mathrm{F}$ & A. sinensis (China) & EtOH & [38] \\
\hline 126 & Aquilarabietic acid G & A. sinensis (China) & $\mathrm{EtOH}$ & [38] \\
\hline 127 & Aquilarabietic acid $\mathrm{H}$ & A. sinensis (China) & EtOH & [38] \\
\hline 128 & Aquilarabietic acid I & A. sinensis (China) & EtOH & [38] \\
\hline 129 & Aquilarabietic acid J & A. sinensis (China) & $\mathrm{EtOH}$ & [38] \\
\hline 130 & Aquilarabietic acid K & A. sinensis (China) & EtOH & [38] \\
\hline 131 & Aquilarin B & A. sinensis (China) & EtOH-EtOAc & [39] \\
\hline 132 & Aquilanol A & A. malaccensis (Laos) & $\mathrm{EtOH}-\mathrm{Et}_{2} \mathrm{O}$ & [36] \\
\hline
\end{tabular}


Table 1. Cont.

\begin{tabular}{|c|c|c|c|c|}
\hline No. & Compound Class and Name & Source or Origin & Extraction * & Ref. \\
\hline \multicolumn{5}{|c|}{ Terpenoids } \\
\hline 133 & Aquilanol B & A. malaccensis (Laos) & $\mathrm{EtOH}-\mathrm{Et}_{2} \mathrm{O}$ & [36] \\
\hline 134 & Daphnauranol D & A. malaccensis (Laos) & $\mathrm{EtOH}-\mathrm{Et}_{2} \mathrm{O}$ & [36] \\
\hline 135 & Chamaejasmone $\mathrm{E}$ & A. malaccensis (Laos) & $\mathrm{EtOH}-\mathrm{Et}_{2} \mathrm{O}$ & [36] \\
\hline 136 & Aquilacallane A & A. sinensis (China) & EtOH-EtOAc & [40] \\
\hline 137 & Aquilacallane B & A. sinensis (China) & EtOH-EtOAc & [40] \\
\hline 138 & Aquimavitalin & A. malaccensis (Taiwan) & EtOH-EtOAc & [41] \\
\hline 139 & 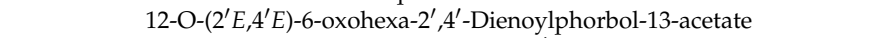 & A. malaccensis (Taiwan) & EtOH-EtOAc & [42] \\
\hline 140 & 12-Deoxy-13-O-acetylphorbol-20-(9' $\left.9^{\prime} Z\right)$-octadecenoate & A. malaccensis (Taiwan) & EtOH-EtOAc & [42] \\
\hline 141 & 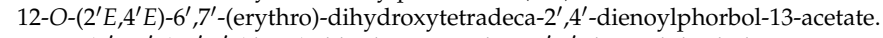 & A. malaccensis (Taiwan) & EtOH-EtOAc & [42] \\
\hline 142 & 12-O-( $\left(2^{\prime} E, 4^{\prime} E\right)-6^{\prime}, 7^{\prime}$-(threo)-dihydroxytetradeca-2', $4^{\prime}$-dienoylphorbol-13-acetate. & A. malaccensis (Taiwan) & EtOH-EtOAc & [42] \\
\hline \multicolumn{5}{|c|}{ Flavonoids } \\
\hline 143 & 4'-O-Geranyltricin & A. sinensis (Taiwan) & EtOH-EtOAc & [27] \\
\hline 144 & $3^{\prime}-\mathrm{O}$-Geranylpolloin & A. sinensis (Taiwan) & EtOH-EtOAc & [27] \\
\hline 145 & Aquisiflavoside & A. sinensis (China) & EtOH- $n-\mathrm{BuOH}$ & [43] \\
\hline 146 & Aquilarisinin & A. sinensis (China) & EtOH- $n-\mathrm{BuOH}$ and EtOAc & [44] \\
\hline 147 & Aquilarisin & A. sinensis (China) & EtOH- $n-\mathrm{BuOH}$ and EtOAc & [44] \\
\hline 148 & Aquilarixanthone & A. sinensis (China) & EtOH- $n-\mathrm{BuOH}$ and EtOAc & [44] \\
\hline 149 & Hypolaetin $5-O-\beta-D$-glucuronopyranoside & A. sinensis (China) & EtOH- $n$-BuOH and EtOAc & [44] \\
\hline 150 & 7- $\beta$-D-Glucoside of 5-O-methylapigenin & A. sinensis (China) & EtOH- $n-\mathrm{BuOH}$ & [45] \\
\hline \multicolumn{5}{|c|}{ Others } \\
\hline 151 & Aquilarinoside $\mathrm{A}$ & A. sinensis (China) & $\mathrm{EtOH}-n-\mathrm{BuOH}$ & [45] \\
\hline 152 & Aquilarin A & A. sinensis (China) & EtOH-EtOAc & [46] \\
\hline 153 & (9S) Megastigma-4,7-diene-2,3,9-triol-9-O- $\beta$-D-glucopyranoside & A. sinensis (China) & $\mathrm{EtOH}-n-\mathrm{BuOH}$ & [47] \\
\hline 154 & (9S) Megastigma-4(13),7-diene-3,6,9-triol-9-O- $\beta$-D-glucopyranoside & A. sinensis (China) & $\mathrm{EtOH}-n-\mathrm{BuOH}$ & [47] \\
\hline
\end{tabular}

* The first one or two solvents used to extract before the separation on columns. Ethanol: EtOH; ethyl acetate: EtOAc; $n$-butyl alcohol: $n$-BuOH; diethyl ether: Et $\mathrm{O}_{2}$; chloroform: $\mathrm{CHCl}_{3}$; and dichloromethane: $\mathrm{CH}_{2} \mathrm{Cl}_{2}$. 


\subsection{Pharmacological Activity of Fraction and Components from Agarwood and Aquilaria Trees}

\subsubsection{Neural Activity}

Agarwood has been traditionally used as a medicine for tranquilizing and reducing excitement in China, Southeast Asia, and the Middle East for centuries. Modern pharmacological studies have demonstrated that agarwood has an active effect on the nervous system [48,49]. Okugawa et al. [50] determined that a benzene extract of $A$. malaccensis agarwood reduced spontaneous motility, prolonged hexobarbiturate-induced sleeping time, and decreased rectal temperature, whereas petroleum ether, chloroform, or water extracts did not have that effect. A further bio-guided isolation of a benzene extract found that jinkoh-eremol and agarospirol were the main active constituents [51,52]. Takemoto et al. [53] reported that agarwood essential oil sedated mice through vapor inhalation, in which the main volatile constituents were benzylacetone, $\alpha$-gurjunene, and $(+)$-calarene. As benzylacetone had a sedative effect, a number of derivatives were synthesized and assessed for a sedative effect. The results demonstrated that benzylacetone-like compounds had sedative activities, and their intensities varied depending on the functional group in the carbon chain, the substituent in the benzene ring, and their combinations [54]. Our recent studies showed that both the ethanol extract and essential oil of agarwood, induced by the whole-tree agarwood inducing technique in A. sinensis trees, had a sedative-hypnotic effect, where its potential mechanism is related to regulating the gene expression of $\mathrm{GABA}_{\mathrm{A}}$ receptors and potentiating the $\mathrm{GABA}_{\mathrm{A}}$ receptor function $[55,56]$. Agarofuran, derived from agarwood essential oil, was reported to have anxiolytic and anti-depression activity in mice [49]. To explore a potential drug for treating anxiety and depression, a series of agarofuran-like derivatives were synthesized and the activity screened, among which, buagafuran was an effective compound for anti-anxiety and anti-depression, with low toxicity and a high safety coefficient $[49,57]$. The potential mechanism might be through modulating central neurotransmitters, such as dopamine [58]. A metabolic study showed that buagafuran could be transformed to hydroxy metabolite and carbonyl one in a human liver microsome, where carbonyl metabolite was the main one [59]. Until now, phase II clinical trials are being conducted on buagafuran. Furthermore, many other activity screening results have also shown that compounds from agarwood have an effect on neural activity. Compound $7(10 \mu \mathrm{g} / \mathrm{mL})$ showed neural protective activity against both glutamate-induced and corticosterone-induced neurotoxicity in PC12 pheochromocytoma and human U251 glioma cells [16]. Compounds 118 and 119 exhibited potent anti-depressant activity in vitro by inhibiting $\left[{ }^{3} \mathrm{H}\right]-5-\mathrm{HT}$ reuptake in rat synaptosomes [37]. Compound $\mathbf{1 2 0}$ demonstrated remarkable antidepressant activity in vitro, by inhibiting norepinephrine reuptake in rat brain synaptosomes [38]. Simultaneously, seventeen new 2-(2-phenylethyl)chromones, including compounds 22, 27-29, 31-33, $\mathbf{6 8}, \mathbf{6 9}, \mathbf{7 8 - 8 0}, \mathbf{8 2}-\mathbf{8 6}$, and eleven new terpenoids, such as 103-105 and 110-117, had acetylcholinesterase inhibitive effect $[8,20,21,26,28,32-35]$. Above all, neural activity of agarwood is one of the most studied aspects with many active compounds and a promising drug candidate found, which will sustain it as a research hotspot in the future.

\subsubsection{Gastrointestinal Regulation}

Pharmacological studies showed that agarwood and the leaves of $A$. sinensis trees have a gastrointestinal regulating effect. Our studies demonstrated that the agarwood ethanol extract significantly improved intestinal peristalsis, enhanced gastric emptying, and inhibited gastric ulcer [60]. $\mathrm{Li}$ et al. reported that the ethanol extract of agarwood and $A$. sinensis leaves enhanced intestinal propulsion [61]. Kakina et al. reported that leaves of $A$. sinensis trees induced laxation via acetylcholine receptors on loperamide-induced constipation in mice [62]. The acetone extract of $A$. sinensis leaves had a laxative effect without causing diarrhea, in which genkwanin $5-O-\beta$-primeveroside was the active constituent, whereas the methanol extract did not have the laxative effect [63]. The ethanol extract of $A$. sinensis leaves had a laxative effect without causing diarrhea in a rat model of low-fiber diet-induced constipation [64]. Mangiferin and genkwanin 5-O-primeveroside were the two major 
bioactive compounds [65]. Additionally, benzylacetone, an active compound from essential oil, had the effect of enhancing appetite [66,67]. Even though agarwood on alleviating abdominal discomfort has been widely used for centuries, the gastrointestinal regulating effect, especially on a specific disease, is not completely clear.

\subsubsection{Antibacterial and Antifungal}

The original use of agarwood was for anticorrosive deodorization in ancient China, as well as Southeast Asian countries. In Thailand, agarwood has been used for a long time as a traditional treatment for infectious diseases such as diarrhea and skin diseases [68]. Chen et al. [69] found that agarwood essential oil derived from A. sinensis, regardless of whether it originated from artificial or natural agarwood, had inhibitive activities towards Bacillus subtilis and Staphylococcus aureus [69]. Extracts of agarwood (A. crassna), isolated by water distillation, supercritical fluid carbon dioxide, and supercritical fluid carbon dioxide with ethanol as the co-solvent, showed antimicrobial activities against $S$. aureus and Candida albicans, but were not against Escherichia coli [70]. Sirilak et al. [68] found that an aqueous extract of A. crassna leaves possessed an in vitro antibacterial action against Staphylococcus epidermidis, causing bacterial cells to swell and distort, inhibiting the biofilm formation, and leading to cell wall rupture. An ethyl acetate soluble fraction of ethanol extract from A. crassna exhibited stronger antifungal (Fusarium solani) activity than ethanol extract [10]. Additionally, many other compounds had an antibacterial activity, such as compound 27, exhibiting inhibitory effect against S. aureus [8], compound 105 and 107 against both $S$. aureus and R. solanacearum, and compound 109 against S. aureus [33]. Even though the antibacterial/antifungal effect of agarwood is definite, the inhibited microbial species are not completely known. Therefore, antibacterial spectrum investigation of agarwood should be carried out.

\subsubsection{Anti-Inflammatory}

Agarwood essential oil has an anti-inflammatory function, significantly reducing the skin thickness, ear weight, oxidative stress, and pro-inflammatory cytokines production in the 12-O-tetradecanoylphorobol-13 acetate (TPA)-induced mouse ear inflammation model [71]. The ethanol extract of agarwood also inhibited ear edema induced by xylene, and peritoneal inflammation induced by low concentrative acetic acid in mice [72]. Linalool and the corresponding acetate derivate play a major role in anti-inflammatory activity [73]. An in silico molecular docking study suggests that 10-epi- $\gamma$-eudesmol, jinkoh-eremol, and agarospirol were preferentially more active than other identified compounds, with strong binding affinity to major anti-inflammatory receptors [71]. Furthermore, many other activity screening results have shown that compounds from agarwood exhibited a potent inhibitory activity against inflammation. Compounds 34-42, 43, 48-51, 52-56, 58, 61-63, 95, 99, and 145 showed significant inhibition of NO production [22-25,30,31,43]. Compound 150 showed inhibition activity against polymorphonuclear neutrophil respiratory burst stimulated by phorbol 12-myristate 13-acetate [45]. Compounds 81 and 144 exhibited inhibition of superoxide anion generation [27], and inversely, compounds 139-142 exerted enhancing activity on superoxide anion generation [42]. At the same time, compounds 81, 139, and 144 showed potent inhibitory activity on elastase release [27,42]. As we all know, inflammation has a close relationship with other diseases, such as immunopathy, metabolic disorders, and neoplasms, so the anti-inflammatory effect of agarwood, in a certain degree, portends the extensive pharmacological activities of agarwood.

\subsubsection{Analgesic Effect}

Wang et al. [74] found that chloroform extracts of agarwood prolonged the pain threshold induced by hot plate, and reduced the times of writhing reactions. Jinkoh-eremol and agarospirol may be the active compounds, and jinkoh-eremol's analgesic effect could be blocked by naloxone (a opioid antagonist), whereas agarosporol was weakly effected by naloxone [51]. At the same time, jinkoh-eremol and agarospirol could inhibit $\mathrm{D}_{2}$ receptor binding and 5- $\mathrm{HT}_{2 \mathrm{~A}}$ receptor binding [51]. 
Additionally, compound 138 showed strong inhibitory activity in A23178- and antigen-induced degranulation assay, with $\mathrm{IC}_{50}$ values of $1.7 \mathrm{nM}$ and $11 \mathrm{nM}$, respectively [41].

\subsubsection{Antiasthma}

The antiasthma effect of agarwood has been traditionally used in China, and can be found in the latest Chinese Pharmacopoeia [13]. However, to our knowledge, only one study found that an ethanol extract of agarwood and $A$. sinensis leaves could inhibit asthma induced by histamine phosphate in guinea pig [75].

\subsubsection{Cytotoxicity}

Agarwood essential oil possesses anticancer activity towards MCF-7 breast cancer cells [76] and HCT 116 colorectal carcinoma cells [77-79]. $\beta$-Caryophyllene, isolated from the essential oil of A. crassna, exhibited selective anti-proliferative effects against colorectal cancer cells $\left(\mathrm{IC}_{50}\right.$ $19 \mu \mathrm{M})$ and induced apoptosis via nuclear condensation and fragmentation pathways. Additionally, $\beta$-caryophyllene also showed potent inhibition of clonogenicity, migration, invasion, and spheroid formation in colon cancer cells [80]. Additionally, other activity screening results showed that compounds from agarwood exhibited cytotoxic activity [81], whereas compound 88 suppressed tumor promotion at noncytotoxic concentrations [29].

\subsubsection{Anti-Diabetes}

Mei et al. [82] found that the ethanol extracts of both agarwood and A. sinensis leaves alleviated diabetes induced by mesoxyalyurea in mice. The methanol extract of $A$. sinensis leaves possessed the fast blood glucose activity in rat and glucose uptake transportation by rat adipocytes [83]. Iriflophenone 3 -C- $\beta$-glucoside decreased the fasting blood glucose levels in streptozocin-induced diabetic mice, and enhanced glucose uptake into adipocytes [84]. Compounds 146-149 isolated from agarwood had an inhibitive effect on $\alpha$-glucosidase [44].

\subsubsection{Antioxidation}

The essential oil of agarwood had a protective effect against oxidative damage induced by hydrogen peroxide $\left(\mathrm{H}_{2} \mathrm{O}_{2}\right)$ in PC12 cells [85]. The aqueous extract of $A$. crassna leaves had radical scavenging capacities determined by $2,2^{\prime}$-azino-bis(3-ethylbenzthiazoline-6-sulphonic acid (ABTS), ferric reducing antioxidant power (FRAP), and 2,2-diphenyl-1-picrylhydrazyl hydrate (DPPH) scavenging assays [68]. A methanol extract of $A$. crassna leaves was also found to have anti-oxidative activities [86]. The 100\% (v/v) ethanol extract exhibited the highest DPPH radical scavenging activity among the $0 \%$ to $100 \%(v / v)$ ethanol extracts isolated from $A$. crassna young leaves [87]. $\beta$-Caryophyllene displayed strong antioxidant effects determined by the DPPH and FRAP scavenging methods [80]. Other compounds $\mathbf{2 8}, \mathbf{3 5}$, and $\mathbf{1 4 4}$, isolated from agarwood, also showed an anti-oxidative effect $[8,22,27]$.

\subsubsection{Others}

A methanol extract of A. crassna leaves significantly reduced fever (rectal temperature) induced by baker's yeast at five and six hours after subcutaneous injection in rat [86]. The aqueous extract of $A$. malaccensis leaves was effective on Trypanosoma evansi with an $\mathrm{IC}_{50}$ value $36.29 \pm 1.32 \mu \mathrm{g} / \mathrm{mL}$, whereas the ethanol extract was relatively weak $\left(\mathrm{IC}_{50}=128.63 \pm 6.70 \mu \mathrm{g} / \mathrm{mL}\right)$ [88]. An ethyl acetate extract of A. crassna showed an anti-ischemic effect by attenuation of P38-MAPK activation [89].

\section{Conclusions}

Among the 154 new compounds identified from Aquilaria plants, 2-(2-phenylethyl)- $4 \mathrm{H}$ chromen-4-one derivatives and sesquiterpenes account for $57 \%$ and $35 \%$, respectively, where most 
of the new compounds, accounting for $89 \%$, were isolated from A. sinensis. Generally, agarwood originating from different Aquilaria plants share some common compounds, but still have several different compounds [14]. In addition, there are at least 19 species of Aquilaria plants producing agarwood, which means that large quantities of new compounds need to be explored in agarwood and Aquilaria plants. The chemical components of agarwood are diverse and complex, contributing to the diversity of bioactivity and pharmacology, including neural activity, gastrointestinal regulation, antibacterial, anti-inflammation, and cytotoxicity. Based on the specific disease and target, illuminating the active ingredients and compounds of agarwood should be carried out, which may not only contribute to the understanding of the scientific nature of the traditional agarwood application, but also benefit the new drug research and agarwood product development.

Acknowledgments: This work was supported by Science \& Technology Programs from Hainan Province of China (No. ZDKJ2016004), the Central Organization Department Plan of the Leading Talents Project of Science and Technology Innovation (No. 99950534), the National Natural Science Foundation of China (Nos. 81403055 and 81303312), Program for Creative Research Groups of Hainan Provincial Natural Science Foundation (No. 2017CXTD022) and the 2016 annual Graduate Innovation Fund Project of PUMC.

Conflicts of Interest: The authors declare no conflict of interest.

\section{References}

1. CITES. Amendments to appendices I and II of CITES. In Proceedings of the Thirteenth Meeting of the Conference of the Parties 2004, Bangkok, Thailand, 2 October 2004.

2. CITES. Government of India Hosted Asian Regional Workshop on the Management of Wild and Planted Agarwood Taxa. Available online: https://www.Cites.Org/eng/2015_india_agarwood_workshop (accessed on 5 May 2015).

3. CITES. Report on NDF of Agarwood for Sustainability Harvest in Indonesia. Available online: https:// cites.Org/sites/default/files/ndf_material/agarwood_in_indonesia_ndf\%5b1\%5d.Pdf (accessed on 29 May 2015).

4. Liu, Y.; Wei, J.; Gao, Z.; Zhang, Z.; Lyu, J. A review of quality assessment and grading for agarwood. Chin. Herb. Med. 2017, 9, 22-30. [CrossRef]

5. The IUCN Red List of Threatened Species. Version 2017-3. Available online: www.Iucnredlist.Org (accessed on 17 December 2017).

6. Kalra, R.; Kaushik, N. A review of chemistry, quality and analysis of infected agarwood tree (Aquilaria sp.). Phytochem. Rev. 2017, 16, 1045-1079. [CrossRef]

7. Liu, Y.; Chen, H.; Yang, Y.; Zhang, Z.; Wei, J.; Meng, H.; Chen, W.; Feng, J.; Gan, B.; Chen, X.; et al. Whole-tree agarwood-inducing technique: An efficient novel technique for producing high-quality agarwood in cultivated Aquilaria sinensis trees. Molecules 2013, 18, 3086-3106. [CrossRef] [PubMed]

8. Li, W.; Cai, C.H.; Dong, W.H.; Guo, Z.K.; Wang, H.; Mei, W.L.; Dai, H.F. 2-(2-phenylethyl)chromone derivatives from Chinese agarwood induced by artificial holing. Fitoterapia 2014, 98, 117-123. [CrossRef] [PubMed]

9. Kalita, J.; Bhattacharyya, P.R.; Boruah, H.P.D.; Unni, B.G.; Lekhak, H.; Nath, S.C. Association of zeuzera conferta walker on agarwood formation in Aquilaria malaccensis Lamk. Asian J. Plant Sci. Res. 2015, 5, 4-9.

10. Novriyanti, E.; Santosa, E.; Syafii, W.; Turjaman, M.; Sitepu, I.R. Antifungal activity of wood extract of Aquilaria crassna Pierre ex Lecomte against agarwood-inducing fungi, Fusarium solani. J. For. Res. 2010, 7, 155-165.

11. Mohamed, R.; Jong, P.L.; Kamziah, A.K. Fungal inoculation induces agarwood in young Aquilaria malaccensis trees in the nursery. J. For. Res. 2014, 25, 201-204. [CrossRef]

12. Peng, C.S.; Osman, M.F.; Bahar, N.; Nuri, E.A.K.; Zakaria, R.; Rahim, K.A. Agarwood inducement technology: A method for producing oil grade agarwood in cultivated Aquilaria malaccensis Lamk. J. Agrobiotechnol. 2015, 6, 1-16.

13. National Pharmacopoeia Committee. Pharmacopoeia of the People's Republic of China; 2015 Version; Chinese Medical Science and Technology Press: Beijing, China, 2015; Volume 1, pp. 185-186.

14. Chen, H.Q.; Wei, J.H.; Yang, J.S.; Zhang, Z.; Yang, Y.; Gao, Z.H.; Sui, C.; Gong, B. Chemical constituents of agarwood originating from the endemic genus Aquilaria plants. Chem. Biodivers. 2012, 9, 236-250. [CrossRef] [PubMed] 
15. Hashim, Y.Z.; Kerr, P.G.; Abbas, P.; Mohd, S.H. Aquilaria spp. (agarwood) as source of health beneficial compounds: A review of traditional use, phytochemistry and pharmacology. J. Ethnopharmacol. 2016, 189, 331-360. [CrossRef] [PubMed]

16. Yang, L.; Qiao, L.; Xie, D.; Yuan, Y.; Chen, N.; Dai, J.; Guo, S. 2-(2-phenylethyl)chromones from Chinese eaglewood. Phytochemistry 2012, 76, 92-97. [CrossRef] [PubMed]

17. Dai, H.F.; Liu, J.; Zeng, Y.B.; Han, Z.; Wang, H.; Mei, W.L. A new 2-(2-phenylethyl)chromone from Chinese eaglewood. Molecules 2009, 14, 5165-5168. [CrossRef] [PubMed]

18. Gao, Y.H.; Liu, J.M.; Lu, H.X.; Wei, Z.X. Two new 2-(2-phenylethyl)chromen-4-ones from Aquilaria sinensis (Lour.) Gilg. Helv. Chim. Acta 2012, 95, 951-954. [CrossRef]

19. Wu, B.; Kwon, S.; Hwang, G.S.; Park, J.H. Eight new 2-(2-phenylethyl)chromone (=2-(2-phenylethyl)-4H1-benzopyran-4-one) derivatives from Aquilaria malaccensis agarwood. Helv. Chim. Acta 2012, 95, 1657-1665. [CrossRef]

20. Liao, G.; Mei, W.L.; Dong, W.H.; Li, W.; Wang, P.; Kong, F.D.; Gai, C.J.; Song, X.Q.; Dai, H.F. 2-(2-phenylethyl)chromone derivatives in artificial agarwood from Aquilaria sinensis. Fitoterapia 2016, 110, 38-43. [CrossRef] [PubMed]

21. Xiang, P.; Mei, W.; Chen, H.; Kong, F.; Wang, H.; Liao, G.; Zhou, L.; Dai, H. Four new bi-phenylethylchromones from artificial agarwood. Fitoterapia 2017, 120, 61-66. [CrossRef] [PubMed]

22. Chen, D.; Xu, Z.; Chai, X.; Zeng, K.; Jia, Y.; Bi, D.; Ma, Z.; Tu, P. Nine 2-(2-phenylethyl)chromone derivatives from the resinous wood of Aquilaria sinensis and their inhibition of LPS-induced NO production in RAW264.7 cells. Eur. J. Org. Chem. 2012, 27, 5389-5397. [CrossRef]

23. Liu, Y.Y.; Chen, D.L.; Wei, J.H.; Feng, J.; Zhang, Z.; Yang, Y.; Zheng, W. Four new 2-(2-phenylethyl)chromone derivatives from Chinese agarwood produced via the whole-tree agarwood-inducing technique. Molecules 2016, 21, 1433. [CrossRef] [PubMed]

24. Huo, H.X.; Gu, Y.F.; Sun, H.; Zhang, Y.F.; Liu, W.J.; Zhu, Z.X.; Shi, S.P.; Song, Y.L.; Jin, H.W.; Zhao, Y.F.; et al. Anti-inflammatory 2-(2-phenylethyl)chromone derivatives from Chinese agarwood. Fitoterapia 2017, 118, 49-55. [CrossRef] [PubMed]

25. Huo, H.X.; Zhu, Z.X.; Song, Y.L.; Shi, S.P.; Sun, J.; Sun, H.; Zhao, Y.F.; Zheng, J.; Ferreira, D.; Zjawiony, J.K.; et al. Anti-inflammatory dimeric 2-(2-phenylethyl)chromones from the resinous wood of Aquilaria sinensis. J. Nat. Prod. 2017. [CrossRef] [PubMed]

26. Liao, G.; Mei, W.L.; Kong, F.D.; Li, W.; Yuan, J.Z.; Dai, H.F. 5,6,7,8-tetrahydro-2-(2-phenylethyl)chromones from artificial agarwood of Aquilaria sinensis and their inhibitory activity against acetylcholinesterase. Phytochemistry 2017, 139, 98-108. [CrossRef] [PubMed]

27. Wang, S.L.; Hwang, T.L.; Chung, M.I.; Sung, P.J.; Shu, C.W.; Cheng, M.J.; Chen, J.J. New flavones, a 2-(2-phenylethyl)-4H-chromen-4-one derivative, and anti-inflammatory constituents from the stem barks of Aquilaria sinensis. Molecules 2015, 20, 20912-20925. [CrossRef] [PubMed]

28. Yang, D.L.; Mei, W.L.; Zeng, Y.B.; Guo, Z.K.; Zhao, Y.X.; Wang, H.; Zuo, W.J.; Dong, W.H.; Wang, Q.H.; Dai, H.F. 2-(2-phenylethyl)chromone derivatives in Chinese agarwood "qi-nan" from Aquilaria sinensis. Planta Med. 2013, 79, 1329-1334. [CrossRef] [PubMed]

29. Suzuki, A.; Miyake, K.; Saito, Y.; Rasyid, F.A.; Tokuda, H.; Takeuchi, M.; Suzuki, N.; Ichiishi, E.; Fujie, T.; Goto, M.; et al. Phenylethylchromones with in vitro antitumor promoting activity from Aquilaria filaria. Planta Med. 2017, 83, 300-305. [CrossRef] [PubMed]

30. Huo, H.X.; Zhu, Z.X.; Pang, D.R.; Li, Y.T.; Huang, Z.; Shi, S.P.; Zheng, J.; Zhang, Q.; Zhao, Y.F.; Tu, P.F.; et al. Anti-neuroinflammatory sesquiterpenes from Chinese eaglewood. Fitoterapia 2015, 106, 115-121. [CrossRef] [PubMed]

31. Zhao, H.; Peng, Q.; Han, Z.; Yang, L.; Wang, Z. Three new sesquiterpenoids and one new sesquiterpenoid derivative from Chinese eaglewood. Molecules 2016, 21, 281. [CrossRef] [PubMed]

32. Yang, D.L.; Wang, H.; Guo, Z.K.; Li, W.; Mei, W.L.; Dai, H.F. Fragrant agarofuran and eremophilane sesquiterpenes in agarwood 'qi-nan' from Aquilaria sinensis. Phytochem. Lett. 2014, 8, 121-125. [CrossRef]

33. Li, W.; Cai, C.H.; Guo, Z.K.; Wang, H.; Zuo, W.J.; Dong, W.H.; Mei, W.L.; Dai, H.F. Five new eudesmane-type sesquiterpenoids from Chinese agarwood induced by artificial holing. Fitoterapia 2015, 100, 44-49. [CrossRef] [PubMed]

34. Yang, D.L.; Li, W.; Dong, W.H.; Wang, J.; Mei, W.L.; Dai, H.F. Five new 5,11-epoxyguaiane sesquiterpenes in agarwood "qi-nan" from Aquilaria sinensis. Fitoterapia 2016, 112, 191-196. [CrossRef] [PubMed] 
35. Li, W.; Liao, G.; Dong, W.H.; Kong, F.D.; Wang, P.; Wang, H.; Mei, W.L.; Dai, H.F. Sesquiterpenoids from Chinese agarwood induced by artificial holing. Molecules 2016, 21, 274. [CrossRef] [PubMed]

36. Ma, C.T.; Eom, T.; Cho, E.; Wu, B.; Kim, T.R.; Oh, K.B.; Han, S.B.; Kwon, S.W.; Park, J.H. Aquilanols A and B, macrocyclic humulene-type sesquiterpenoids from the agarwood of Aquilaria malaccensis. J. Nat. Prod. 2017, 80, 3043-3048. [CrossRef] [PubMed]

37. Yang, L.; Qiao, L.R.; Zhang, J.J.; Dai, J.G.; Guo, S.X. Two new sesquiterpene derivatives from Chinese eaglewood. J. Asian Nat. Prod. Res. 2012, 14, 1054-1058. [CrossRef] [PubMed]

38. Yang, L.; Qiao, L.; Ji, C.; Xie, D.; Gong, N.B.; Lu, Y.; Zhang, J.; Dai, J.; Guo, S. Antidepressant abietane diterpenoids from Chinese eaglewood. J. Nat. Prod. 2013, 76, 216-222. [CrossRef] [PubMed]

39. Peng, K.; Mei, W.L.; Zhao, Y.X.; Tan, L.H.; Wang, Q.H.; Dai, H.F. A novel degraded sesquiterpene from the fresh stem of Aquilaria sinensis. J. Asian Nat. Prod. Res. 2011, 13, 951-955. [CrossRef] [PubMed]

40. Cheng, J.T.; Han, Y.Q.; He, J.; De Wu, X.; Dong, L.B.; Peng, L.Y.; Li, Y.; Zhao, Q.S. Two new tirucallane triterpenoids from the leaves of Aquilaria sinensis. Arch. Pharm. Res. 2013, 36, 1084-1089. [CrossRef] [PubMed]

41. Korinek, M.; Wagh, V.D.; Lo, I.W.; Hsu, Y.M.; Hsu, H.Y.; Hwang, T.L.; Wu, Y.C.; Cheng, Y.B.; Chen, B.H.; Chang, F.R. Antiallergic phorbol ester from the seeds of Aquilaria malaccensis. Int. J. Mol. Sci. 2016, 17, 398. [CrossRef] [PubMed]

42. Wagh, V.D.; Korinek, M.; Lo, I.W.; Hsu, Y.M.; Chen, S.L.; Hsu, H.Y.; Hwang, T.L.; Wu, Y.C.; Chen, B.H.; Cheng, Y.B.; et al. Inflammation modulatory phorbol esters from the seeds of Aquilaria malaccensis. J. Nat. Prod. 2017, 80, 1421-1427. [CrossRef] [PubMed]

43. Yang, X.B.; Feng, J.; Yang, X.W.; Zhao, B.; Liu, J.X. Aquisiflavoside, a new nitric oxide production inhibitor from the leaves of Aquilaria sinensis. J. Asian Nat. Prod. Res. 2012, 14, 867-872. [CrossRef] [PubMed]

44. Feng, J.; Yang, X.W.; Wang, R.F. Bio-assay guided isolation and identification of $\alpha$-glucosidase inhibitors from the leaves of Aquilaria sinensis. Phytochemistry 2011, 72, 242-247. [CrossRef] [PubMed]

45. Qi, J.; Lu, J.J.; Liu, J.H.; Yu, B.Y. Flavonoid and a rare benzophenone glycoside from the leaves of Aquilaria sinensis. Chem. Pharm. Bull. (Tokyo) 2009, 57, 134-137. [CrossRef] [PubMed]

46. Wang, Q.H.; Peng, K.; Tan, L.H.; Dai, H.F. Aquilarin A, a new benzenoid derivative from the fresh stem of Aquilaria sinensis. Molecules 2010, 15, 4011-4016. [CrossRef] [PubMed]

47. Sun, J.; Xia, F.; Wang, S.; Wang, K.Y.; Chen, J.M.; Tu, P.F. Structural elucidation of two new megastigmane glycosides from the leaves of Aquilaria sinensis. Chin. J. Nat. Med. 2015, 13, 290-294. [PubMed]

48. Chinese Academy of Medical Sciences the Institute of Materia Medica. Modern Research of Chinese Herbal Medicine; Version 1; China Union Medical University Press: Beijing, China, 2010; Volume 3, pp. 156-158.

49. Guo, J.; Wang, W.; Fang, H.; Liu, Q.; Zhang, W. United State Patent: Agarofuan Derivatives, Their Preparation, Pharmaceutical Composition Containing Them and Their Use as Medicine. U.S. Patent 6486201b1, 26 November 2002.

50. Okugawa, H.; Ueda, R.; Matsumoto, K.; Kawanishi, K.; Kato, A. Effects of agarwood extracts on the central nervous system in mice. Planta Med. 1993, 59, 32-36. [CrossRef] [PubMed]

51. Okugawa, H.; Ueda, R.; Matsumoto, K.; Kawanishi, K.; Kato, K. Effects of sesquiterpenoids from "oriental incenses" on acetic acid-induced writhing and $\mathrm{D}_{2}$ and 5- $\mathrm{HT}_{2 \mathrm{a}}$ receptors in rat brain. Phytomedicine 2000, 7, 417-422. [CrossRef]

52. Okugawa, H.; Ueda, R.; Matsumoto, K.; Kawanishi, K.; Kato, A. Effect of jinkoh-eremol and agarospirol from agarwood on the central nervous system in mice. Planta Med. 1996, 62, 2-6. [CrossRef] [PubMed]

53. Takemoto, H.; Ito, M.; Shiraki, T.; Yagura, T.; Honda, G. Sedative effects of vapor inhalation of agarwood oil and spikenard extract and identification of their active components. J. Nat. Med. 2008, 62, 41-46. [CrossRef] [PubMed]

54. Miyosh, T.; Ito, M.; Kitayama, T.; Isomori, S.; Yamashit, F. Sedative effects of inhaled benzylacetone and structural features contributing to its activity. Biol. Pharm. Bull. 2013, 36, 1474-1481. [CrossRef]

55. Wang, S.; Wang, C.; Peng, D.; Liu, X.; Wu, C.; Guo, P.; Wei, J. Agarwood essential oil displays sedative-hypnotic effects through the GABAergic system. Molecules 2017, 22, 2190. [CrossRef] [PubMed]

56. Wang, S.; Zhou, Y.; Ma, F.; Zhang, Q.; Liu, Y.; Gong, B.; Guo, P.; Wei, J. Effect of agarwood produced by whole-tree agarwood-inducing technique on hypnotic and spontaneous activity inhibition of mice. J. Int. Pharm. Res. 2016, 43, 1082-1087.

57. Liu, Q.; Wang, D.; Li, C.; Lv, D. The synthensis and centrol nervous system activity of agarofuran. Chin. J. Med. Chem. 2003, 13, 125-130. 
58. Zhang, Y.; Wang, W.; Zhang, J. Effects of novel anxiolytic 4-butyl-alpha-agarofuran on levels of monoamine neurotransmitters in rats. Eur. J. Pharmacol. 2004, 504, 39-44. [CrossRef] [PubMed]

59. Li, N.; Zhang, J.; Zhou, T. Metabolic research of new antidepression drug AF-5 and its metabolin in human liver microsomes in vitro. Yao Xue Xue Bao 2001, 36, 528-531. [PubMed]

60. Liu, Y.; Wang, S.; Zhou, Y.; Zhang, Q.; Ma, F.; Gong, B.; Guo, P.; Wei, J. Effect of agarwood extracts produced by the whole-tree agarwood-inducing technique on gastrointestinal motility and gastric ulcer. J. Int. Pharm. Res. 2016, 43, 1076-4081.

61. Li, H.; Jiang, Z.; Mei, Q. Comparasion the intestinal propulsion effect of agarwood leaves and resion. Asia Pac. Tradit. Med. 2013, 9, 24-25.

62. Kakino, M.; Izuta, H.; Ito, T.; Tsuruma, K.; Araki, Y.; Shimazawa, M.; Oyama, M.; Iinuma, M.; Hara, H. Agarwood induced laxative effects via acetylcholine receptors on loperamide-induced constipation in mice. Biosci. Biotechnol. Biochem. 2010, 74, 1550-1555. [CrossRef] [PubMed]

63. Hara, H.; Ise, Y.; Morimoto, N.; Shimazawa, M.; Ichihashi, K.; Ohyama, M.; Iinuma, M. Laxative effect of agarwood leaves and its mechanism. Biosci. Biotechnol. Biochem. 2008, 72, 335-345. [CrossRef] [PubMed]

64. Kakino, M.; Tazawa, S.; Maruyama, H.; Tsuruma, K.; Araki, Y.; Shimazawa, M.; Hara, H. Laxative effects of agarwood on low-fiber diet-induced constipation in rats. BMC Complement. Altern. Med. 2010, 10, 68. [CrossRef] [PubMed]

65. Ito, T.; Kakino, M.; Tazawa, S.; Watara, T.; Oyama, M.; Maruyama, H. Quantification of polyphenols and pharmacological analysis of water and ethanol-based extracts of cultivated agarwood leaves. J. Nutr. Sci. Vitaminol. 2012, 58, 136-142. [CrossRef] [PubMed]

66. Ogawa, K.; Ito, M. Appetite-enhancing effects of trans-cinnamaldehyde, benzylacetone and 1-phenyl-2-butanone by inhalation. Planta Med. 2016, 82, 84-88. [CrossRef] [PubMed]

67. Ogawa, K.; Ito, M. Appetite-enhancing effects: The influence of concentrations of benzylacetone and trans-cinnamaldehyde and their inhalation time, as well as the effect of aroma, on body weight in mice. Biol. Pharm. Bull. 2016, 39, 794-798. [CrossRef] [PubMed]

68. Kamonwannasit, S.; Nantapong, N.; Kumkrai, P.; Luecha, P.; Kupittayanant, S.; Chudapongse, N. Antibacterial activity of Aquilaria crassna leaf extract against Staphylococcus epidermidis by disruption of cell wall. Ann. Clin. Microbiol. Antimicrob. 2013, 12, 20. [CrossRef] [PubMed]

69. Chen, H.; Yang, Y.; Xue, J.; Wei, J.; Zhang, Z.; Chen, H. Comparison of compositions and antimicrobial activities of essential oils from chemically stimulated agarwood, wild agarwood and healthy Aquilaria sinensis (Lour.) Gilg trees. Molecules 2011, 16, 4884-4896. [CrossRef] [PubMed]

70. Wetwitayaklung, P.; Thavanapong, N.; Pharmacognosy, D.O.; Charoenteeraboon, J. Chemical constituents and antimicrobial activity of essential oil and extracts of heartwood of Aquilaria crassna obtained from water distillation and supercritical fluid carbon dioxide extraction. Silpakorn Univ. Sci. Technol. J. 2009, 3, 25-33.

71. Yadav, D.K.; Mudgal, V.; Agrawal, J.; Maurya, A.J.; Bawankule, D.U.; Chanotiya, C.S.; Khan, F.; Thul, S.T. Molecular docking and ADME studies of natural compounds of agarwood oil for topical anti-inflammatory activity. Curr. Comput. Aided Drug Des. 2013, 9, 360-370. [CrossRef] [PubMed]

72. Lin, Z.; Li, H.; Mei, Q. Comparative study on antiinflammatory of agarwood leaves and resion. Chin. Ache. Tradit. Chin. Med. 2013, 31, 548-549.

73. Peana, A.T.; D'Aquila, P.S.; Panin, F.; Serra, G.; Pippia, P.; Moretti, M.D. Anti-inflammatory activity of linalool and linalyl acetate constituents of essential oils. Phytomedicine 2002, 9, 721-726. [CrossRef] [PubMed]

74. Wang, J.; Xu, X.; Liang, Y. Comparative study on analgesic effects of different years of Chinese eaglewood. Hainan Med. J. 2014, 25, 2188-2190.

75. Wu, X.; Li, H.; Mei, Q.; Lin, Z. Comparative study on preventing asthma effects of agarwood leaves and resion. Pharm. Today 2013, 23, 346-347.

76. Hashim, Y.; Phirdaous, A.; Azura, A. Screening of anticancer activity from agarwood essential oil. Pharmacogn. Res. 2014, 6, 191-194. [CrossRef] [PubMed]

77. Dahham, S.S.; Hassan, L.E.; Ahamed, M.B.; Majid, A.S.; Majid, A.M.; Zulkepli, N.N. In vivo toxicity and antitumor activity of essential oils extract from agarwood (Aquilaria crassna). BMC Complement. Altern. Med. 2016, 16, 236. [CrossRef] [PubMed]

78. Dahham, S.; Tabana, Y.; Sandai, D.; Ahmed, M. In vitro anticancer and antiangiogenic activity of essential oils extracts from agarwood Aquilaria crassna. Med. Aromat. Plants 2016, 5, 256-268. [CrossRef] 
79. Ibrahim, A.; Rawi, S.; Abdul, M.; Rahman, N.N.A.; Salah, K.M.A.; AbKadir, M.O. Separation and fractionation of Aquilaria malaccensis oil using supercritical fluid extraction and the cytotoxic properties of the extracted oil. Procedia Food Sci. 2011, 1, 1953-1959. [CrossRef]

80. Dahham, S.S.; Tabana, Y.M.; Iqbal, M.A.; Ahamed, M.B.; Ezzat, M.O.; Majid, A.S.; Majid, A.M. The anticancer, antioxidant and antimicrobial properties of the sesquiterpene $\beta$-caryophyllene from the essential oil of Aquilaria crassna. Molecules 2015, 20, 11808-11829. [CrossRef] [PubMed]

81. Liu, J.; Wu, J.; Zhao, Y.X.; Deng, Y.Y.; Mei, W.L.; Dai, H.F. A new cytotoxic 2-(2-phenylethyl)chromone from Chinese eaglewood. Chin. Chem. Lett. 2008, 19, 934-936. [CrossRef]

82. Mei, Q.; Li, H.; Lin, Z.; Wu, X.; Liang, L. Comparative study on antidiabetes of agarwood leaves and resion. Lishizhen Med. Mater. Med. Res. 2013, 24, 1606-1607.

83. Pranakhon, R.; Aromdee, C. Antihyperglycemic activity of agarwood leaf extracts in STZ-induced diabetic rats and glucose uptake enhancement activity in rat adipocytes. Songklanakarin J. Sci. Technol. 2011, 33, 405-410.

84. Pranakhon, R.; Aromdee, C.; Pannangpetch, P. Effects of iriflophenone 3-c- $\beta$-glucoside on fasting blood glucose level and glucose uptake. Pharmacogn. Mag. 2015, 11, 82-89. [PubMed]

85. Xiong, L.; Li, L.; Lin, L.; Chen, D.; Wu, J. Protective effect of lignum Aquilaria resionatum essential oil on $\mathrm{H}_{2} \mathrm{O}_{2}$-induced oxidative damage of PC12 cells. Tradit. Chin. Drug Res. Clin. Pharm. 2014, 25, $28-32$.

86. Sattayasai, J.; Bantadkit, J.; Aromdee, C.; Lattmann, E.; Airarat, W. Antipyretic, analgesic and anti-oxidative activities of Aquilaria crassna leaves extract in rodents. J. Ayurveda Integr. Med. 2012, 3, 175-179. [CrossRef] [PubMed]

87. Tay, P.; Tan, C.; Abas, F.; Yim, H.; Ho, C. Assessment of extraction parameters on antioxidant capacity, polyphenol content, epigallocatechin gallate (EGCG), epicatechin gallate (ECG) and iriflophenone 3-c- $\beta$-glucoside of agarwood (Aquilaria crassna) young leaves. Molecules 2014, 19, 12304-12319. [CrossRef] [PubMed]

88. Dyary, H.O.; Arifah, A.K.; Sharma, R.S.; Rasedee, A.; Mohd-Aspollah, M.S.; Zakaria, Z.A.; Zuraini, A.; Somchit, M.N. Antitrypanosomal screening and cytotoxic effects of selected medicinal plants. Trop. Biomed. 2014, 31, 89-96. [PubMed]

89. Suwannasing, C. Anti-ischemic effect of ethyl acetate extract of Aquilaria crassna by attenuation of p38-MAPK activation. J. Appl. Pharm. Sci. 2012, 2, 26-30. [CrossRef] 